\title{
On the structure of the tensor square of the natural module of the symmetric group
}

\author{
Jürgen Müller and Johannes Orlob
}

\begin{abstract}
We determine the submodule structure of the tensor square of the natural module of the symmetric group over a field of prime characteristic. To do so, we also determine the submodule structure of certain Young modules over a field of characteristic 2 .

Mathematics Subject Classification: 20C30, 20C20.

Keywords: Symmetric group, natural module, Young module, tensor square, submodule lattice.
\end{abstract}

\section{Introduction and results}

One of the central problems of the modular representation theory of the symmetric group $\mathcal{S}_{n}$ is to give a precise description of its simple modules over fields of prime characteristic $p$. When it comes to their properties, astonishingly little is known about the structure of the tensor product of two such simple modules. It is even an open problem when precisely such a tensor product is simple again; conjecturally [11] this only happens under very restrictive circumstances in characteristic 2 , but although major steps have been taken $[4,12]$ this has not yet been settled affirmatively.

In the present paper we consider the natural module $D^{[n-1,1]}$, which is the nontrivial constituent of the natural permutation module of $\mathcal{S}_{n}$. We determine the direct summands and the submodule structure of the tensor square $D^{[n-1,1]} \otimes$ $D^{[n-1,1]}$, the results being presented in (1.2) and (1.4) for $p=2$ and $p$ odd, respectively. Since the prime field $\mathbb{F}_{p}$ is a splitting field of $\mathcal{S}_{n}$ we restrict ourselves to this case, and in particular the tensor product is understood over $\mathbb{F}_{p}$. Many of the results for the case $p$ odd have already been obtained in [28], where also detailed conjectures for the case $p=2$ have been made. The latter case turns out to be much more complicated than the former one, and to proceed we exploit the close relationship of the submodule structure of the tensor square to that of the Young modules labelled by the partitions $[n-2,2]$ and $\left[n-2,1^{2}\right]$.

Although Young modules play a prominent role in the modular representation theory of $\mathcal{S}_{n}$, and the Young modules labelled by the two lexicographically largest partitions $[n]$ and $[n-1,1]$ are well-understood anyway, there are only a few results in the literature, being commented on below, on the submodule lattices of the Young modules labelled by the next largest partitions $[n-2,2]$ and $\left[n-2,1^{2}\right]$. Hence we first determine the submodule structure of the latter modules; while this is almost immediate for $p$ odd, these results for $p=2$ might be of independent interest as well. 
Table 1: Hasse diagrams of Young modules $Y_{\mathbb{F}_{2}}^{[n-2,2]}$ for $p=2$ and $n \geq 5$.
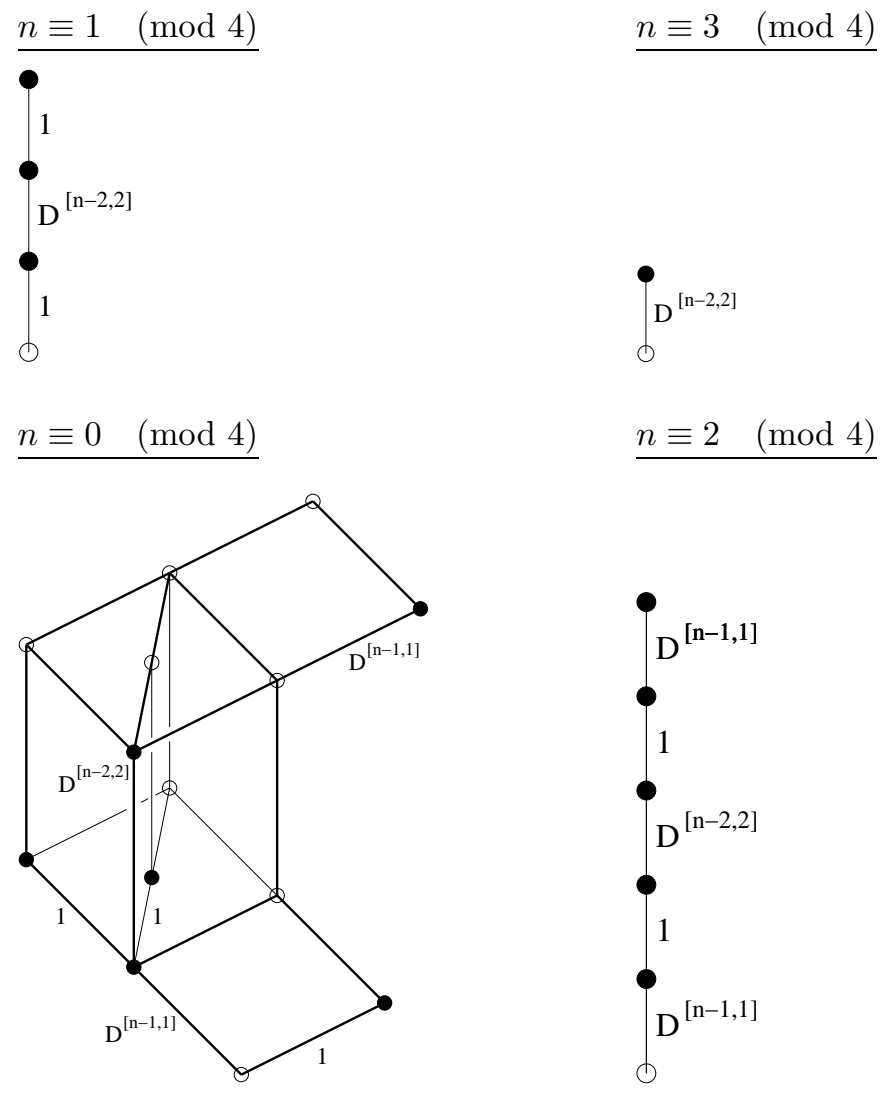

(1.1) Theorem. a) The submodule lattices of the Young modules over $\mathbb{F}_{2}$ labelled by $[n-2,2]$ where $n \geq 4$, and $\left[n-2,1^{2}\right]$ where $n \geq 3$, are as given in Tables 1-3.

b) The submodule lattices of the non-simple Young modules over $\mathbb{F}_{p}$ labelled by $[n-2,2]$ and $\left[n-2,1^{2}\right]$, where $p$ is odd and $n \geq 4$, are as given in Table 4 .

In the Hasse diagrams shown, the local submodules, see (2.7), are depicted as filled vertices. We only indicate their types, since these suffice to determine the isomorphism types of all simple subquotients. Amongst the above cases only the submodule lattices of the Young modules labelled by $[n-2,2]$ for $p=2$ and $n \equiv 0(\bmod 4)$ are not distributive, but there is a unique dotted-line, see (2.7), consisting of local submodules of type $1_{\mathbb{F}_{2}}$. In the distributive cases the submodule lattices remain unchanged under extension $\mathbb{F}_{p} \subseteq F$ of the base field, while in the non-distributive cases the cardinality of the dotted-line increases, 
Table 2: Hasse diagrams of Young modules $Y_{\mathbb{F}_{2}}^{\left[n-2,1^{2}\right]}$ for $p=2$ and $n \geq 5$.

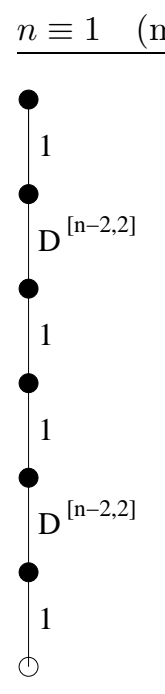

$$
\underline{n \equiv 3 \quad(\bmod 4)}
$$

$$
\underline{n \equiv 0 \quad(\bmod 4)}
$$
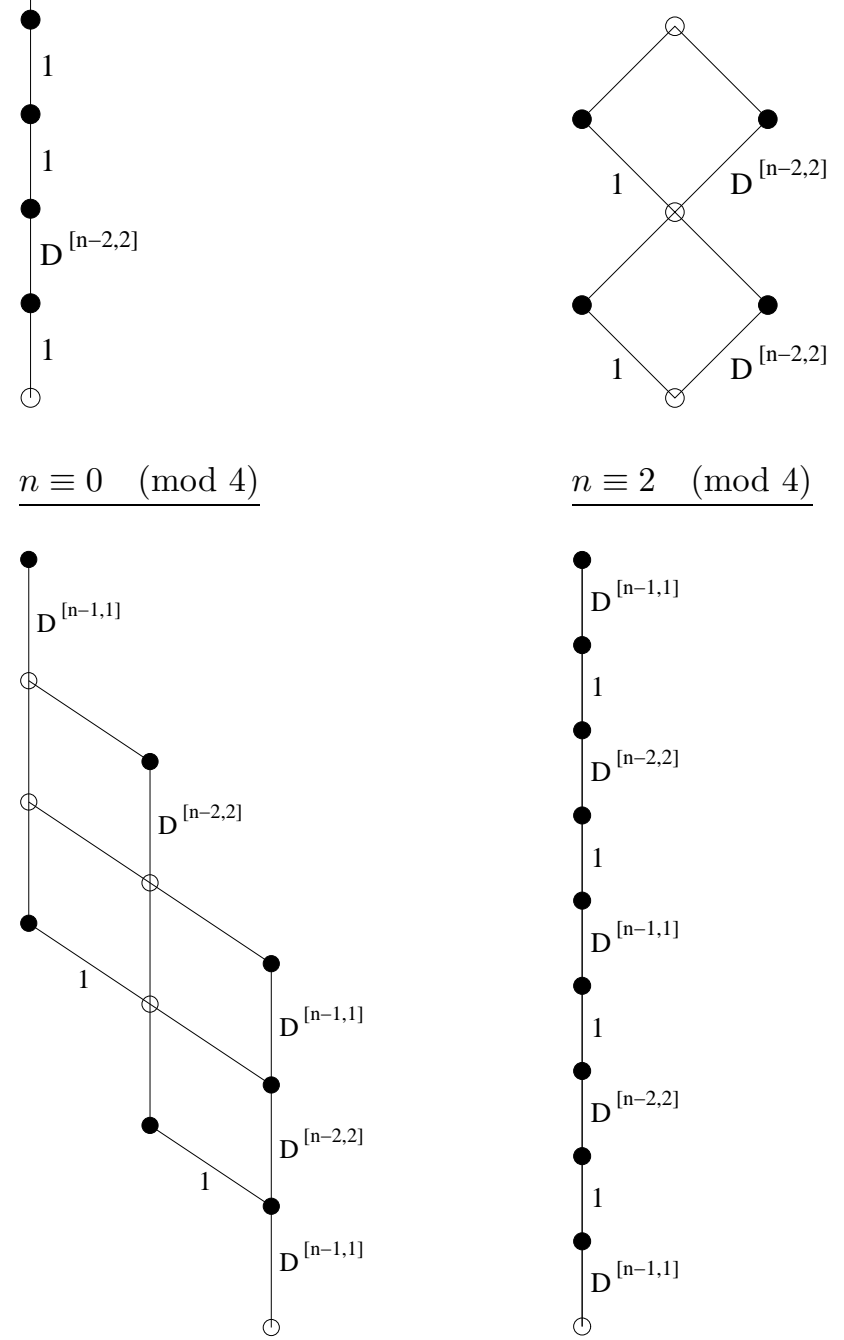

$n \equiv 2 \quad(\bmod 4)$

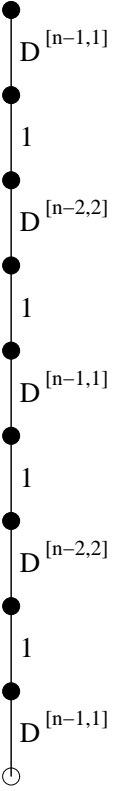


Table 3: Hasse diagrams of Young modules for $p=2$ and $n \in\{3,4\}$.

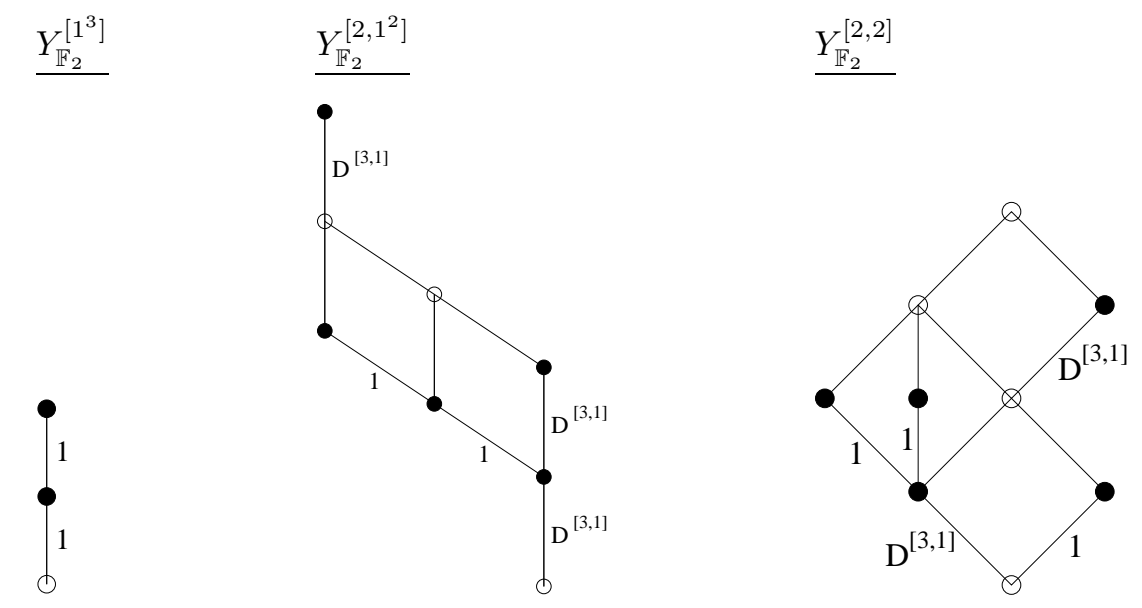

as the latter is in bijection with $F \dot{\cup}\{\infty\}$.

A few comments on the results available in the literature are in order. As for the 2-part partition case $[n-2,2]$, the associated Young modules have been dealt with for $p$ odd in [4, La.1.2] and [6, La.3.3], and for $p=2$ and $n$ odd in [4, La.1.3]. Moreover, by [15, Thm.6.4] the Young modules labelled by $[n-2,2]$ and $[m-2,2]$, respectively, have isomorphic submodule lattices whenever $m \geq n$ such that $(n, p) \neq(4,2)$ and $m \equiv n(\bmod 2)$ and $m \equiv n\left(\bmod p^{\min \left\{e \in \mathbb{N} ; p^{e}>n\right\}}\right)$. Although these congruences do not reflect all lattice isomorphisms, for fixed $p$ we are reduced to a finite number of cases, which could be examined explicitly by the computational techniques mentioned in (1.5), but we prefer to avoid this involved machinery and give a straightforward proof for $p=2$ and $n$ even.

As for the partition $\left[n-2,1^{2}\right]$ and $p=2$, the induced Specht module $\left(S_{\mathbb{F}_{2}}^{[n-2,1]}\right)^{\mathcal{S}_{n}}$ is examined amongst others in [17, Sect.6]. For $n$ even the latter coincides with the Young module $Y_{\mathbb{F}_{2}}^{\left[n-2,1^{2}\right]}$, while for $n$ odd its principal block component is a submodule of the Young module with quotient module $1_{\mathbb{F}_{2}}$. Still, the results in [17, Diagram V] do not seem to help to determine the submodule lattice of the Young module, but conversely our results imply that, using the notation of [17], for $n$ even we have $b \sim S_{\mathbb{F}_{2}}^{\left[n-2,1^{2}\right]}, c \sim D^{[n-1,1]}$ and $a, d, e \sim 0$, while for $n$ odd we have $b \sim S_{\mathbb{F}_{2}}^{[n-2,2]}, a \sim D^{[n-1,1]}, e \sim 1_{\mathbb{F}_{2}}$ and $c, d \sim 0$.

Having these prerequisites in place, we are prepared to state the results on the submodule structure of the tensor square $D^{[n-1,1]} \otimes D^{[n-1,1]}$. 
Table 4: Hasse diagrams of non-simple Young modules for $p$ odd and $n \geq 4$.
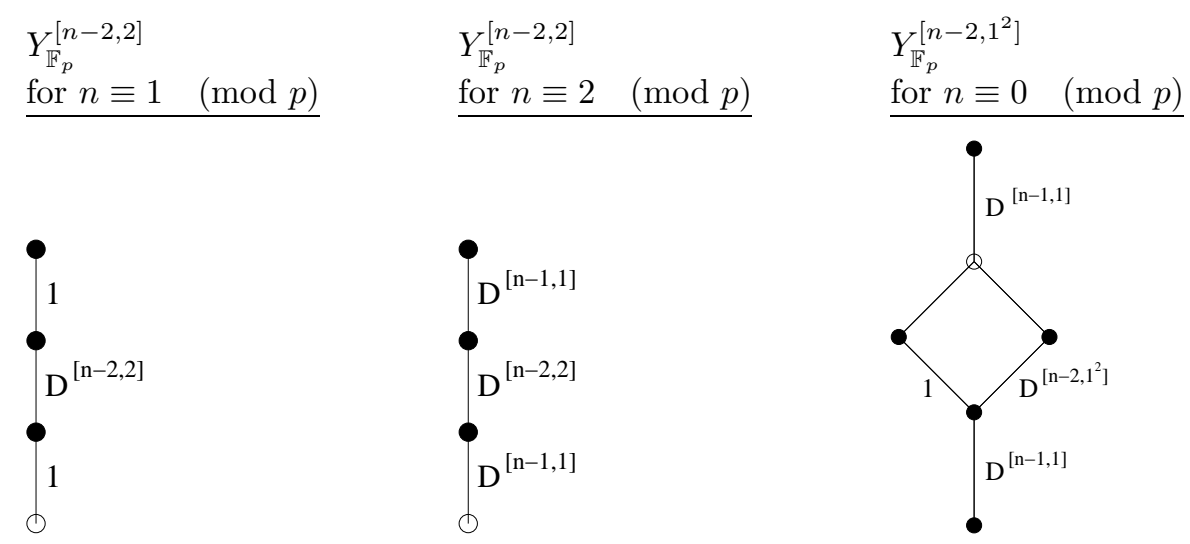

$(\bmod p)$

$\underline{\text { for } n=2(\bmod p)}$

$\underline{\text { for } n \equiv 0-(\bmod p)}$

(1.2) Theorem. Let $p=2$, let $n \geq 3$ and let $D:=D^{[n-1,1]}$.

a) For $n$ odd we have $D \otimes D \cong D \oplus Y_{\mathbb{F}_{2}}^{\left[n-2,1^{2}\right]}$.

b) For $n$ even we have $D \otimes D \cong \operatorname{rad}\left(Y_{\mathbb{F}_{2}}^{\left[n-2,1^{2}\right]}\right) / \operatorname{soc}\left(Y_{\mathbb{F}_{2}}^{\left[n-2,1^{2}\right]}\right)$, and $D \otimes D$ is indecomposable if and only if $n \neq 4$.

Thus the submodule lattice of the tensor square can be read off from the Hasse diagram of the Young module $Y_{\mathbb{F}_{2}}^{\left[n-2,1^{2}\right]}$. Since for $n$ odd $D^{[n-1,1]}$ is not a constituent of $Y_{\mathbb{F}_{2}}^{\left[n-2,1^{2}\right]}$, in this case the submodule lattice of the tensor square is the lattice direct sum of those of $D^{[n-1,1]}$ and $Y_{\mathbb{F}_{2}}^{\left[n-2,1^{2}\right]}$. In any case, the submodule lattice of the tensor square is distributive, and thus remains unchanged under extension of the base field.

(1.3) Corollary. Let $S^{2}(D)$ and $\Lambda^{2}(D)$ denote the symmetric and exterior square of $D:=D^{[n-1,1]}$, respectively. Thus by $[9$, La.11.3] there is a filtration $\{0\}<\Lambda^{2}(D)<S^{2}(D)<D \otimes D$ with layers $D \otimes D \cong\left[\frac{\Lambda^{2}(D)}{D}\right]$, the notation being explained in (2.1). Hence for $n$ odd we have $S^{2}(D) \cong D \oplus \Lambda^{2}(D)$, where for $n \equiv 1(\bmod 4)$ we have $\Lambda^{2}(D) \cong\left[\begin{array}{c}1_{\mathbb{F}_{2}} \\ D^{[n-2,2]} \\ 1_{\mathbb{F}_{2}}\end{array}\right]$, and for $n \equiv 3(\bmod 4)$ we have $\Lambda^{2}(D) \cong 1_{\mathbb{F}_{2}} \oplus D^{[n-2,2]}$. For $n \equiv 0(\bmod 4)$ we have $\Lambda^{2}(D) \cong 1_{\mathbb{F}_{2}} \oplus D^{[n-2,2]}$ 
and $S^{2}(D) \cong 1_{\mathbb{F}_{2}} \oplus\left[\begin{array}{c}D \\ D^{[n-2,2]}\end{array}\right]$, and for $n \equiv 2(\bmod 4)$ we have

$$
\Lambda^{2}(D) \cong\left[\begin{array}{c}
1_{\mathbb{F}_{2}} \\
D^{[n-2,2]} \\
1_{\mathbb{F}_{2}}
\end{array}\right] \quad \text { and } \quad S^{2}(D) \cong\left[\begin{array}{c}
D \\
1_{\mathbb{F}_{2}} \\
D^{[n-2,2]} \\
1_{\mathbb{F}_{2}}
\end{array}\right]
$$

(1.4) Theorem. Let $p$ be an odd prime, let $n \geq 4$ and let $D:=D^{[n-1,1]}$. a) For $n \equiv 0(\bmod p)$ we have

$$
\begin{aligned}
D \otimes D & \cong\left(1_{\mathbb{F}_{p}} \oplus D^{[n-2,2]}\right) \oplus\left(D^{\left[n-2,1^{2}\right]}\right) \\
& \cong D^{[n-2,2]} \oplus \operatorname{rad}\left(Y_{\mathbb{F}_{p}}^{\left[n-2,1^{2}\right]}\right) / \operatorname{soc}\left(Y_{\mathbb{F}_{p}}^{\left[n-2,1^{2}\right]}\right) .
\end{aligned}
$$

b) For $n \equiv 1(\bmod p)$ we have $D \otimes D \cong\left(D \oplus Y_{\mathbb{F}_{p}}^{[n-2,2]}\right) \oplus\left(D^{\left[n-2,1^{2}\right]}\right)$.

c) For $n \equiv 2(\bmod p)$ we have $D \otimes D \cong\left(1_{\mathbb{F}_{p}} \oplus Y_{\mathbb{F}_{p}}^{[n-2,2]}\right) \oplus\left(D^{\left[n-2,1^{2}\right]}\right)$.

d) For $n \not \equiv 0,1,2(\bmod p)$ we have $D \otimes D \cong\left(1_{\mathbb{F}_{p}} \oplus D \oplus D^{[n-2,2]}\right) \oplus\left(D^{\left[n-2,1^{2}\right]}\right)$.

For the case $n=3$ we for $p=3$ have $D^{[2,1]} \otimes D^{[2,1]} \cong 1_{\mathbb{F}_{p}}$, while for $p>3$ we have $D^{[2,1]} \otimes D^{[2,1]} \cong\left(1_{\mathbb{F}_{p}} \oplus D^{[2,1]}\right) \oplus\left(D^{\left[1^{3}\right]}\right)$. Hence for all $n \geq 3$ the submodule lattice of $D \otimes D$ is distributive, and is the lattice direct sum of the submodule lattices of its direct summands.

We have $D \otimes D \cong S^{2}(D) \oplus \Lambda^{2}(D)$, where the bracketed terms in (1.4) indicate the symmetric and exterior squares, respectively. Actually, it is known by folklore that we always have $\Lambda^{2}(D) \cong D^{\left[n-2,1^{2}\right]}$; this is implicit in [29] and an explicit proof is given in [26].

The present paper is organised as follows. In Section 2 we fix the notation, and to make the paper sufficiently self-contained we in (2.2)-(2.6) recall the necessary auxiliary facts from the representation theory of the symmetric group. In (2.7) we introduce some notions from the structure theory of combinatorial lattices, in particular local submodules and dotted-lines which are the building blocks of modular lattices. After these preparations, the submodule structure of the Young modules mentioned in Table 4, i. e. for $p$ odd, is immediate, while in Sections 3 and 4 we proceed to examine the Young modules mentioned in Tables 1 and 2 of (1.1), respectively. Here, the strategy employed is as follows. For the uniserial cases we directly head towards building up the unique composition series, which coincides with both the Loewy series and the socle series. For the other cases we determine the local submodules, and where existent the dottedlines. The submodule lattices can then be determined purely combinatorially as described in (2.7). In Section 5 we finally prove (1.2) and (1.4). 
(1.5) Computations. To get some feeling how the patterns governing the structure of the modules under consideration might look like, prior to attempting general proofs we have computed a bunch of explicit examples [28], using techniques from computational representation theory. In particular, we thus have found the results in Table 3 and the submodule lattice of $Y_{\mathbb{F}_{2}}^{\left[3,1^{2}\right]}$ in (4.5).

More precisely, for various partitions $\lambda \vdash n$, for some smallish $n$, the computer algebra system GAP [10] was used to construct the permutation action of $\mathcal{S}_{n}$ on the cosets of the Young subgroup $\mathcal{S}_{\lambda}<\mathcal{S}_{n}$. Using the computer algebra system MeatAxe [27], dealing with matrix representations over finite fields, and its facilities to compute direct sum decompositions [31, 22], the Young module labelled by $\lambda$ was computed as a direct summand of the permutation module associated with $\mathcal{S}_{\lambda}$. Using the facilities to compute submodule lattices [21] also available in the MeatAxe, the submodule lattice of the Young module was then determined. Similarly, the natural module $D^{[n-1,1]}$ was constructed as a constituent of the $p$-modular reduction of the natural permutation action of $\mathcal{S}_{n}$, and the direct summands of the tensor square $D^{[n-1,1]} \otimes D^{[n-1,1]}$ and their submodule lattices have been computed.

\section{Preliminaries}

(2.1) a) Prerequisites. We assume the reader familiar with the representation theory of the symmetric group $[18,19]$, in particular with the modular branching rule for restriction of simple modules in positive characteristic [20, Thm.11.2.7], and with the fact that Specht modules in positive characteristic have Jantzen filtrations with self-dual layers which give rise to the JantzenSchaper formula, [2] and [24, Cor.5.33]. Moreover, we assume the reader familiar with the notion of Young lattices, $[16,13,8]$ and [23, Ch.4.6], in particular with the fact that Young lattices are self-dual and have Specht filtrations which are governed by the dominance partial ordering on partitions [23, La.4.6.2, Thm.4.6.4], and with Klyachko's formula for the multiplicities of Young lattices as direct summands in certain permutation lattices, [13, Thm.7.14] and [23, Thm.4.6.3]. Finally, we assume the reader familiar with the basic notions from the theory of combinatorial lattices [5].

b) Notation. All modules considered are finitely generated unital right modules. If $V$ is an $A$-module, where $A$ is a finite dimensional $F$-algebra over a field $F$, and $S$ is a simple $A$-module, then the multiplicity of $S$ in a composition series of $V$ is denoted by $[V: S]$, and if $A$-modules $V$ and $W$ have the same constituents including multiplicities then we write $V \sim W$. If $V$ is uniserial having a composition series with descending simple layers $S, T$ then we depict this as $V \cong\left[\begin{array}{c}S \\ T\end{array}\right]$, while if $V$ has a filtration $\{0\}<W<V$ then we write $V \cong\left[\frac{V / W}{W}\right]$, and we use a similar notation for longer composition series or filtrations. To emphasise that this does not determine $V$ up to isomorphism, we 
Table 5: Permutation modules for $n \geq 4$.

\begin{tabular}{|c|c|c|c|c|c|}
\hline $\operatorname{rk}_{\mathbb{Z}}\left(S^{\mu}\right)$ & $M^{\lambda}$ & $\Sigma$ & $\begin{array}{l}\overrightarrow{7} \\
\text { I } \\
\stackrel{1}{2}\end{array}$ & $\begin{array}{l}\mathrm{N} \\
\mathcal{N} \\
1 \\
\tilde{\Sigma}\end{array}$ & $\begin{array}{c}\underset{N}{\sim} \\
\text { N } \\
\text { I } \\
\tilde{E}\end{array}$ \\
\hline 1 & {$[n]$} & 1 & 1 & 1 & 1 \\
\hline$n-1$ & {$[n-1,1]$} & $\cdot$ & 1 & 1 & 2 \\
\hline$\frac{n(n-3)}{2}$ & {$[n-2,2]$} & . & . & 1 & 1 \\
\hline$\frac{(n-1)(n-2)}{2}$ & {$\left[n-2,1^{2}\right]$} & . & . & . & 1 \\
\hline
\end{tabular}

speak of the shape of $V$ instead. For a $\mathbb{Z}$-lattice $V$ we let $V_{\mathbb{Q}}:=V \otimes_{\mathbb{Z}} \mathbb{Q}$ and $V_{\mathbb{F}_{p}}:=V \otimes_{\mathbb{Z}} \mathbb{F}_{p}$. Similarly, letting $\mathbb{Z}_{(p)} \subseteq \mathbb{Q}$ be the localisation of $\mathbb{Z}$ at the prime ideal $(p) \triangleleft \mathbb{Z}$, for a $\mathbb{Z}_{(p)}$-lattice $V$ we let $V_{\mathbb{Q}}:=V \otimes_{\mathbb{Z}_{(p)}} \mathbb{Q}$ and $V_{\mathbb{F}_{p}}:=V \otimes_{\mathbb{Z}_{(p)}} \mathbb{F}_{p}$.

For a partition $\lambda \vdash n$ let $S^{\lambda}$ be the associated Specht $\mathbb{Z} \mathcal{S}_{n}$-lattice. In particular we let $1_{\mathbb{Z}}:=S^{[n]}$ be the trivial $\mathbb{Z} \mathcal{S}_{n}$-lattice. The simple $\mathbb{F}_{p} \mathcal{S}_{n}$-modules $D^{\lambda}$ are labelled by $p$-row regular partitions $\lambda \vdash n$. Again we let $1_{\mathbb{F}_{p}}:=D^{[n]}$ be the trivial $\mathbb{F}_{p} \mathcal{S}_{n}$-module. For $\lambda \vdash n$ let $M^{\lambda}$ be the permutation $\mathbb{Z} \mathcal{S}_{n}$-lattice induced by the action of $\mathcal{S}_{n}$ on the cosets of the Young subgroup $\mathcal{S}_{\lambda} \leq \mathcal{S}_{n}$, and let $Y^{\lambda} \leq M_{\mathbb{Z}_{(p)}}^{\lambda}$ be the associated Young $\mathbb{Z}_{(p)} \mathcal{S}_{n}$-lattice. The $p$-Kostka number $\left[M_{\mathbb{Z}_{(p)}}^{\lambda}: Y^{\mu}\right]$, i. e. the multiplicity of the Young lattice $Y^{\mu}$, where $\mu \vdash n$, as a direct summand of $M_{\mathbb{Z}_{(p)}}^{\lambda}$ is well-defined by [1, Cor.3.11.4], and [7, Exc.6.16] applied to $\operatorname{End}_{\mathcal{S}_{n}}\left(M_{\mathbb{Z}_{(p)}}^{\lambda}\right)$.

Let $\operatorname{Irr}\left(\mathcal{S}_{n}\right)$ be the set of irreducible ordinary characters of $\mathcal{S}_{n}$, and let $\chi^{\lambda} \in$ $\operatorname{Irr}\left(\mathcal{S}_{n}\right)$ be the character afforded by $S^{\lambda}$. In particular we let $1_{\mathcal{S}_{n}}:=\chi^{[n]}$ and $1_{\mathcal{S}_{n}}^{-}:=\chi^{\left[1^{n}\right]}$ be the trivial and the sign character, respectively. Finally, let $\psi^{\lambda}=1_{\mathcal{S}_{\lambda}}^{\mathcal{S}_{n}} \in \mathbb{Z} \operatorname{Irr}\left(\mathcal{S}_{n}\right)$ be the permutation character afforded by $M^{\lambda}$.

(2.2) Permutation modules. The Kostka numbers $\left[M_{\mathbb{Q}}^{\lambda}: S_{\mathbb{Q}}^{\mu}\right]$ are known $[19$, Ch.2.2]. For $\lambda \in\left\{[n],[n-1,1],[n-2,2],\left[n-2,1^{2}\right]\right\}$ and $n \geq 4$ they are reproduced in Table 5 , where the case $n=3$ is recovered by deleting the row and the column belonging to $[n-2,2]$.

Since the natural permutation action is doubly transitive, the tensor square of the permutation character $\psi^{[n-1,1]}$ equals $\psi^{[n-1,1]} \otimes \psi^{[n-1,1]}=\psi^{[n-1,1]}+$ $\psi^{\left[n-2,1^{2}\right]}$. This for $n \geq 4$ yields $\chi^{[n-1,1]} \otimes \chi^{[n-1,1]}=\left(\chi^{[n]}+\chi^{[n-1,1]}+\chi^{[n-2,2]}\right)+$ $\left(\chi^{\left[n-2,1^{2}\right]}\right)$, while for $n=3$ we have $\chi^{[2,1]} \otimes \chi^{[2,1]}=\left(\chi^{[3]}+\chi^{[2,1]}\right)+\left(\chi^{\left[1^{3}\right]}\right)$; here the bracketed terms indicate the symmetric and exterior squares, respectively.

(2.3) Specht modules. The decomposition multiplicities $\left[S_{\mathbb{F}_{p}}^{\mu}: D^{\lambda}\right]$, where $\mu \in\left\{[n],[n-1,1],[n-2,2],\left[n-2,1^{2}\right]\right\}$ and $n \geq 3$, are reproduced in Table 6 
Table 6: Partial decomposition matrices for $p=2$ and $n \geq 5$.

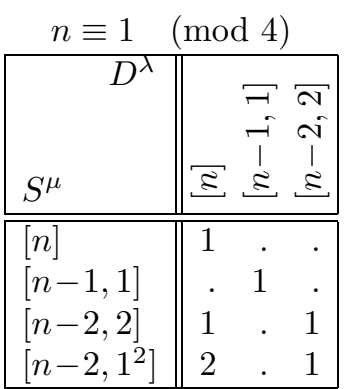

\begin{tabular}{|c|c|c|}
\hline$D^{\lambda}$ & $\Sigma$ & 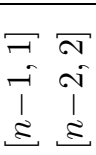 \\
\hline$[n]$ & 1 & . \\
\hline$[n-1,1]$ & . & 1 \\
\hline$[n-2,2]$ & ${ }^{\circ}$ & . 1 \\
\hline$\left[n-2,1^{2}\right]$ & 1 & . 1 \\
\hline
\end{tabular}

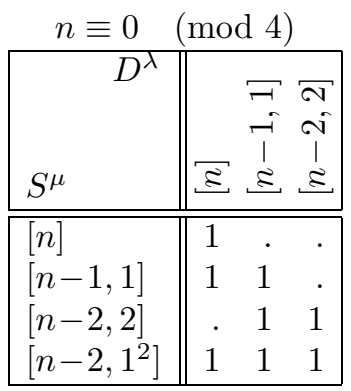

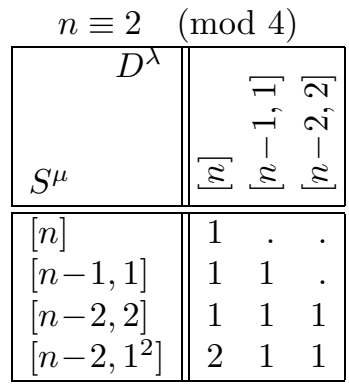

for $p=2$ and in Table 7 for $p$ odd. Here the case $n=3$ is covered by deleting the row and the column belonging to $[n-2,2]$, and for $p=2$ the case $n=4$ is covered by deleting the column belonging to $[n-2,2]$. Note that the partial decomposition matrices already precisely reflect the $p$-block distribution of the various $S_{\mathbb{F}_{p}}^{\mu}$ and $D^{\lambda}$ mentioned.

The decomposition multiplicities for the 2-part partition cases $S_{\mathbb{F}_{p}}^{[n-1,1]}$ and $S_{\mathbb{F}_{p}}^{[n-2,2]}$ are known by [18, Thm.24.15] and [18, p.137], and those of the hook partition case $S_{\mathbb{F}_{p}}^{\left[n-2,1^{2}\right]}$ for $p$ odd by Peel's Theorem, [29] and [18, Thm.24.1]. The remaining case $S_{\mathbb{F}_{2}}^{\left[n-2,1^{2}\right]}$ is contained in [17, Thm.7.1], or we from Table 5 simply observe $\chi^{\left[n-2,1^{2}\right]}-\chi^{[n-2,2]}-\chi^{[n]}=\psi^{\left[n-2,1^{2}\right]}-2 \cdot \psi^{[n-2,2]}=1_{\mathcal{S}_{n-2}}^{\mathcal{S}_{n}}-2 \cdot 1_{\mathcal{S}_{n-2} \times \mathcal{S}_{2}}^{\mathcal{S}_{n}}=$ $\left(1_{\mathcal{S}_{n-2}} \otimes 1_{\mathcal{S}_{2}}^{-}-1_{\mathcal{S}_{n-2}} \otimes 1_{\mathcal{S}_{2}}\right)^{\mathcal{S}_{n}}$, hence restricting to odd order elements we get $\left.\chi^{\left[n-2,1^{2}\right]}\right|_{2^{\prime}}=\left.\left(\chi^{[n]}+\chi^{[n-2,2]}\right)\right|_{2^{\prime}}$.

(2.4) Specht modules in characteristic 2. The Specht modules $S_{\mathbb{F}_{2}}^{\mu}$, where $\mu \in\left\{[n-1,1],[n-2,2],\left[n-2,1^{2}\right]\right\}$ and $n \geq 3$, have the following structure.

a) For $n$ odd we have $S_{\mathbb{F}_{2}}^{[n-1,1]} \cong D^{[n-1,1]}$, for $n$ even $S_{\mathbb{F}_{2}}^{[n-1,1]} \cong\left[\begin{array}{c}D^{[n-1,1]} \\ 1_{\mathbb{F}_{2}}\end{array}\right]$. 
Table 7: Partial decomposition matrices for $p$ odd and $n \geq 4$.

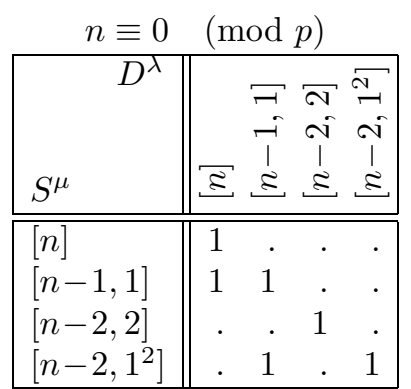

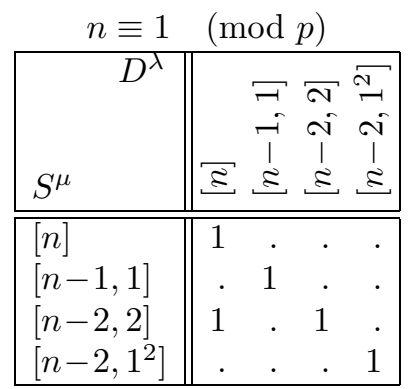

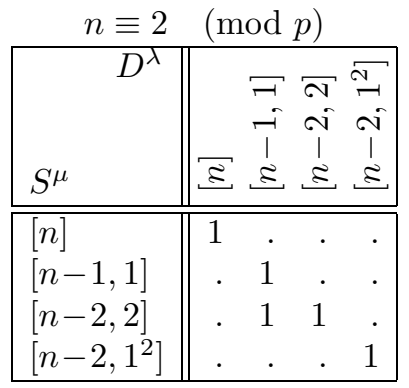

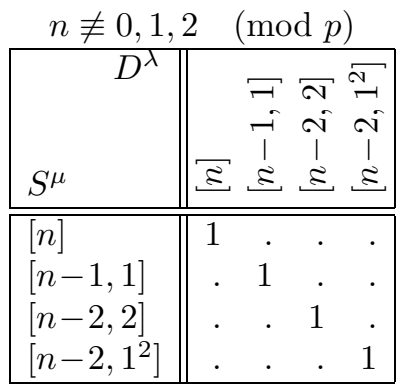

b) For $n \equiv 1(\bmod 4)$ we have $S_{\mathbb{F}_{2}}^{[n-2,2]} \cong\left[\begin{array}{c}D^{[n-2,2]} \\ 1_{\mathbb{F}_{2}}\end{array}\right]$, while for $n \equiv 3$ $(\bmod 4)$ and $n \neq 3$ we have $S_{\mathbb{F}_{2}}^{[n-2,2]} \cong D^{[n-2,2]}$. For $n \equiv 0(\bmod 4)$ we have $S_{\mathbb{F}_{2}}^{[n-2,2]} \cong\left[\begin{array}{l}D^{[n-2,2]} \\ D^{[n-1,1]}\end{array}\right]$, while for $n \equiv 2(\bmod 4)$ we by $[18$, Thm.24.4] have $\operatorname{Hom}_{\mathcal{S}_{n}}\left(1_{\mathbb{F}_{2}}, S_{\mathbb{F}_{2}}^{[n-2,2]}\right)=\{0\}$, implying $S_{\mathbb{F}_{2}}^{[n-2,2]} \cong\left[\begin{array}{c}D^{[n-2,2]} \\ 1_{\mathbb{F}_{2}} \\ D^{[n-1,1]}\end{array}\right]$.

c) For $n$ odd the Jantzen-Schaper formula shows that $S_{\mathbb{F}_{2}}^{\left[n-2,1^{2}\right]}$ has only one Jantzen layer. This for $n \equiv 3(\bmod 4)$ implies $S_{\mathbb{F}_{2}}^{\left[n-2,1^{2}\right]} \cong 1_{\mathbb{F}_{2}} \oplus D^{[n-2,2]}$. For $n \equiv 1(\bmod 4)$ we only infer that $S_{\mathbb{F}_{2}}^{\left[n-2,1^{2}\right]}$ is self-dual; it is shown as a byproduct in (4.2) that $S_{\mathbb{F}_{2}}^{\left[n-2,1^{2}\right]} \cong\left[\begin{array}{c}1_{\mathbb{F}_{2}} \\ D^{[n-2,2]} \\ 1_{\mathbb{F}_{2}}\end{array}\right]$.

For $n$ even the Jantzen-Schaper formula shows that $1_{\mathbb{F}_{2}}$ and $D^{[n-2,2]}$ occur in the first Jantzen layer of $S_{\mathbb{F}_{2}}^{\left[n-2,1^{2}\right]}$, while $D^{[n-1,1]}$ occurs in a higher layer. Hence for $n \equiv 0(\bmod 4)$ we have a filtration $S_{\mathbb{F}_{2}}^{\left[n-2,1^{2}\right]} \cong\left[\frac{1_{\mathbb{F}_{2}} \oplus D^{[n-2,2]}}{D^{[n-1,1]}}\right]$; it is shown 
as a by-product in (4.3) that $\operatorname{rad}\left(S_{\mathbb{F}_{2}}^{\left[n-2,1^{2}\right]}\right)=\operatorname{soc}\left(S_{\mathbb{F}_{2}}^{\left[n-2,1^{2}\right]}\right) \cong D^{[n-1,1]}$. For $n \equiv 2(\bmod 4)$ we have $S_{\mathbb{F}_{2}}^{\left[n-2,1^{2}\right]} \cong\left[\frac{V}{D^{[n-1,1]}}\right]$, where $V \sim 2 \cdot 1_{\mathbb{F}_{2}} \oplus D^{[n-2,2]}$ is self-dual; it is shown as a by-product in (4.4) that $S_{\mathbb{F}_{2}}^{\left[n-2,1^{2}\right]}$ is uniserial.

(2.5) Restrictions of simple modules in characteristic 2. We determine the structure of $\left.D^{[n-1,1]}\right|_{\mathcal{S}_{n-1}}$ and $\left.D^{[n-2,2]}\right|_{\mathcal{S}_{n-1}}$, where $p=2$ and $n \geq 5$. The constituents of the direct summands of the restrictions to $\mathcal{S}_{n-1}$ of the simple modules labelled by 2-part partitions are known by [30, Thm.3.1].

For $n$ even we have $\left.D^{[n-1,1]}\right|_{\mathcal{S}_{n-1}} \cong D^{[n-2,1]}$, for $n$ odd we have $\left.D^{[n-1,1]}\right|_{\mathcal{S}_{n-1}} \cong$ $\left[\begin{array}{c}1_{\mathbb{F}_{2}} \\ D^{[n-2,1]} \\ 1_{\mathbb{F}_{2}}\end{array}\right]$. Moreover, for $n$ even we have $\left.D^{[n-2,2]}\right|_{\mathcal{S}_{n-1}} \cong D^{[n-3,2]}$.

It is slightly more involved to determine the structure of $\left.D^{[n-2,2]}\right|_{\mathcal{S}_{n-1}}$ for $n$ odd. The modular branching rule yields $\operatorname{soc}\left(\left.D^{[n-2,2]}\right|_{\mathcal{S}_{n-1}}\right) \cong D^{[n-2,1]} \cong$ $\left(\left.D^{[n-2,2]}\right|_{\mathcal{S}_{n-1}}\right) / \operatorname{rad}\left(\left.D^{[n-2,2]}\right|_{\mathcal{S}_{n-1}}\right)$. For $n \equiv 1(\bmod 4)$ and $n \neq 5$ this implies $\left.D^{[n-2,2]}\right|_{\mathcal{S}_{n-1}} \cong\left[\begin{array}{l}D^{[n-2,1]} \\ D^{[n-3,2]} \\ D^{[n-2,1]}\end{array}\right]$, while for $n=5$ we get $\left.D^{[3,2]}\right|_{\mathcal{S}_{4}} \cong\left[\begin{array}{c}D^{[3,1]} \\ D^{[3,1]}\end{array}\right]$. For $n \equiv 3(\bmod 4)$ we only infer that $\operatorname{rad}\left(\left.D^{[n-2,2]}\right|_{\mathcal{S}_{n-1}}\right) / \operatorname{soc}\left(\left.D^{[n-2,2]}\right|_{\mathcal{S}_{n-1}}\right) \sim$ $2 \cdot 1_{\mathbb{F}_{2}} \oplus D^{[n-3,2]}$ is self-dual; it is shown in (3.2) and used in (4.4) and (4.8) that $\left.D^{[n-2,2]}\right|_{\mathcal{S}_{n-1}}$ is uniserial such that

$$
\left.D^{[n-2,2]}\right|_{\mathcal{S}_{n-1}} \cong\left[\begin{array}{c}
D^{[n-2,1]} \\
1_{\mathbb{F}_{2}} \\
D^{[n-3,2]} \\
1_{\mathbb{F}_{2}} \\
D^{[n-2,1]}
\end{array}\right]
$$

(2.6) Young modules. The decomposition multiplicities $\left[Y_{\mathbb{Q}}^{\lambda}: S_{\mathbb{Q}}^{\mu}\right]$, where $\lambda \in$ $\left\{[n],[n-1,1],[n-2,2],\left[n-2,1^{2}\right]\right\}$ and $n \geq 3$, are reproduced in Table 8 for $p=2$ and in Table 9 for $p$ odd. Here the case $n=3$ is covered by deleting the row and the column belonging to $[n-2,2]$.

The $p$-Kostka numbers, and hence the decomposition multiplicities, for the 2part partition cases $Y^{[n-1,1]}$ and $Y^{[n-2,2]}$ are known by [14, Thm.3.3]. For $p$ odd $1_{\mathcal{S}_{n-2}}^{\mathcal{S}_{n}}=\left(1_{\mathcal{S}_{n-2}} \otimes 1_{\mathcal{S}_{2}}+1_{\mathcal{S}_{n-2}} \otimes 1_{\mathcal{S}_{2}}^{-}\right)^{\mathcal{S}_{n}}$ implies $M_{\mathbb{Z}_{(p)}}^{\left[n-2,1^{2}\right]} \cong M_{\mathbb{Z}_{(p)}}^{[n-2,2]} \oplus Y^{\left[n-2,1^{2}\right]}$, while for $p=2$ Klyachko's formula implies $M_{\mathbb{Z}_{(2)}}^{\left[n-2,1^{2}\right]} \cong 2 \cdot Y^{[n-1,1]} \oplus Y^{\left[n-2,1^{2}\right]}$ for $n$ odd, and $M_{\mathbb{Z}_{(2)}}^{\left[n-2,1^{2}\right]} \cong Y^{[n-1,1]} \oplus Y^{\left[n-2,1^{2}\right]}$ for $n$ even.

(2.7) Submodule lattices. We need a few notions from the theory of combinatorial lattices which are not readily available in textbooks. Most of this 
Table 8: Young modules for $p=2$ and $n \geq 4$.
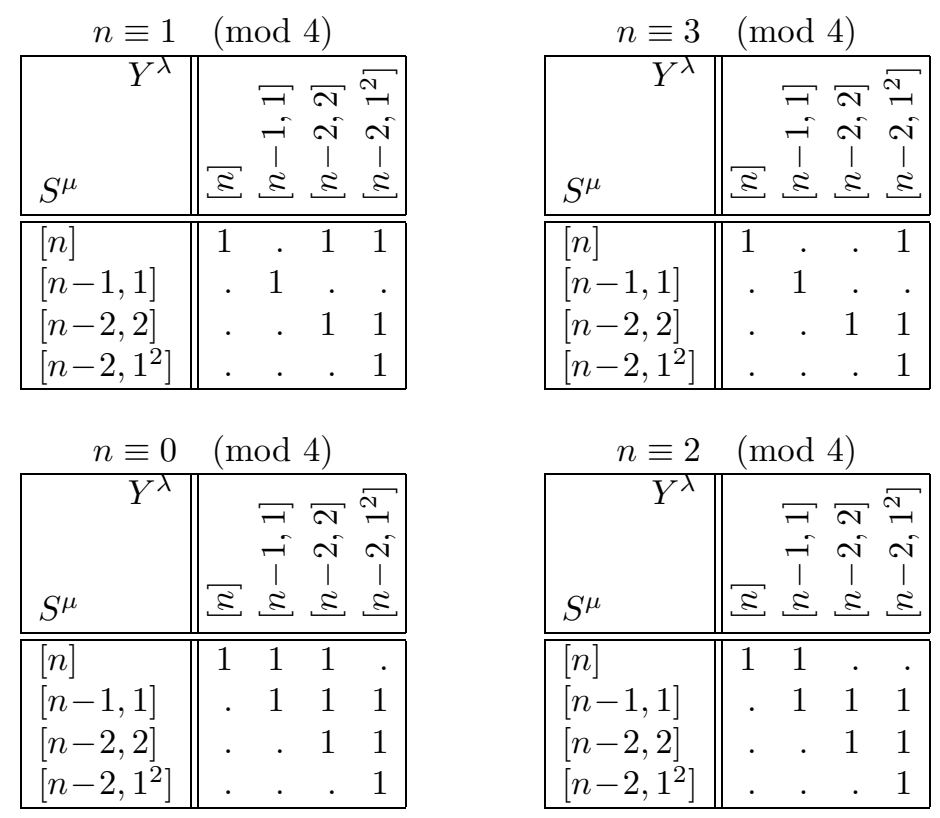

actually holds much more generally for modular lattices [25], but here we restrict ourselves to submodule lattices.

a) Local submodules. Let $A$ be a finite dimensional $F$-algebra, where $F$ is a field, let $V$ be an $A$-module. The set $\mathcal{M}(V)$ of its submodules is a modular lattice of finite length, whose join and meet operations are sum and intersection of submodules, respectively. For any simple $A$-module $S$ let

$$
\begin{aligned}
\mathcal{L}_{S}(V) & :=\{U \leq V ; U / \operatorname{rad}(U) \cong S\} \\
\mathcal{L}_{S}^{2}(V) & :=\{U \leq V ; U / \operatorname{rad}(U) \cong S \oplus S\}, \\
\mathcal{L}_{S}^{\infty}(V) & :=\left\{U \leq V ; U / \operatorname{rad}(U) \cong \bigoplus_{i=1}^{r} S \text { for some } r \in \mathbb{N}_{0}\right\} .
\end{aligned}
$$

The elements of $\mathcal{L}_{S}(V)$ are called the local submodules of type $S$, or the $S$ local submodules for short. The set $\mathcal{L}_{S}^{\infty}(V)$ is a lattice whose join operation still is the sum of submodules, but where the meet of $U, U^{\prime} \in \mathcal{L}_{S}^{\infty}(V)$ is given by $\sum\left\{W \in \mathcal{L}_{S}^{\infty}(V) ; W \leq U \cap U^{\prime}\right\}$. Summing over all simple $A$-modules yields

$$
\begin{aligned}
\mathcal{L}(V) & :=\coprod_{S / \cong} \mathcal{L}_{S}(V)=\{U \leq V ; U / \operatorname{rad}(U) \text { simple }\}, \\
\mathcal{L}^{2}(V) & :=\coprod_{S / \cong} \mathcal{L}_{S}^{2}(V)=\{U \leq V ; U / \operatorname{rad}(U) \cong S \oplus S \text { for some } S\} .
\end{aligned}
$$

In particular, $\mathcal{L}(V)$ is the set of join-irreducible elements of $\mathcal{M}(V)$. These sets can be used to give a description of the full submodule lattice $\mathcal{M}(V)$ as follows. 
Table 9: Young modules for $p$ odd and $n \geq 4$.

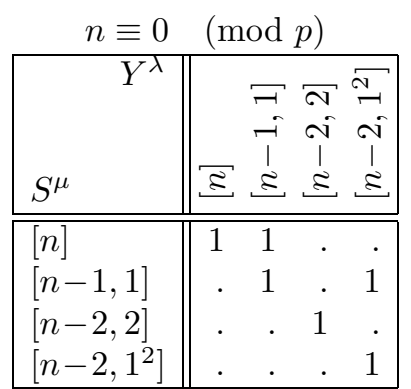

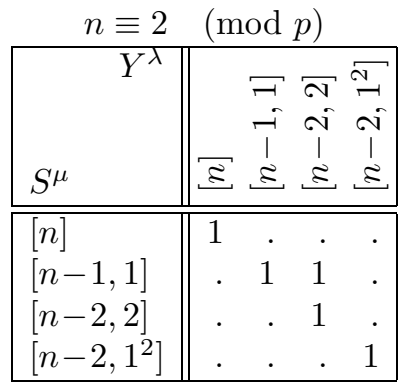

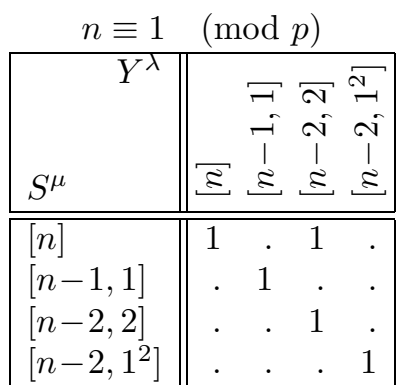

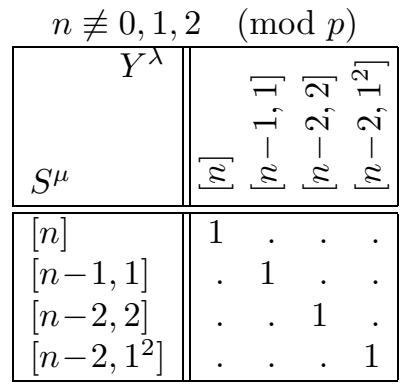

For any $U \in \mathcal{L}_{S}^{2}(V)$ let $\left\{U_{i}<U ; i \in \mathcal{I}_{U}\right\}$ be the set of its maximal submodules, where $\mathcal{I}_{U}$ is a suitable index set. A set $\mathcal{D}_{U}=\left\{L_{i} \in \mathcal{L}_{S}(V) ; i \in \mathcal{I}_{U}\right\}$ such that $L_{i}+\operatorname{rad}(U)=U_{i}$, for all $i \in \mathcal{I}_{U}$, is called a dotted-line for $U$. By [25, Thm.5.2] dotted-lines always exist, and we may identify $\mathcal{I}_{U}=\operatorname{End}_{A}(S) \cup\{\infty\}$, where $\operatorname{End}_{A}(S)$ denotes the endomorphism skew field of $S$, and ' $\infty$ ' is one further element disjoint to $\operatorname{End}_{A}(S)$. In particular we have $\left|\mathcal{I}_{U}\right| \geq 3$.

Now, for any $U \in \mathcal{L}^{2}(V)$ we arbitrarily pick a dotted-line $\mathcal{D}_{U} \subseteq \mathcal{L}(V)$ and keep it fixed. Let $\mathcal{M}(\mathcal{L}(V))$ be the set of subsets $\mathcal{X} \subseteq \mathcal{L}(V)$ such that i) whenever we have $L \in \mathcal{X}$ and $L^{\prime} \in \mathcal{L}(V)$ such that $L^{\prime} \leq L$, then we also have $L^{\prime} \in \mathcal{X}$, and ii) for any $U \in \mathcal{L}^{2}(V)$ and the chosen dotted-line $\mathcal{D}_{U} \subseteq \mathcal{L}(V)$ we either have $\left|\mathcal{D}_{U} \cap \mathcal{X}\right| \leq 1$ or $\mathcal{D}_{U} \subseteq \mathcal{X}$. Generalising the result in [3] we have the following mutually inverse inclusion-preserving bijections [25, Thm.2.5, Thm.2.7]

$$
\begin{array}{ll}
\mathcal{M}(V) \rightarrow \mathcal{M}(\mathcal{L}(V)): & U \mapsto\{L \in \mathcal{L}(V) ; L \leq U\} \\
\mathcal{M}(\mathcal{L}(V)) \rightarrow \mathcal{M}(V): & \mathcal{X} \mapsto \sum \mathcal{X} .
\end{array}
$$

Hence it suffices to determine the local submodules and the pairwise inclusions between them, as well as $\mathcal{L}^{2}(V)$ and the associated dotted-lines, then $\mathcal{M}(V)$ can be determined purely combinatorially using the above bijections. In particular, by [25, Cor.2.8] the lattice $\mathcal{M}(V)$ is distributive if and only if $\mathcal{L}^{2}(V)=\emptyset$, and if $\mathcal{M}(V)$ is distributive and $F$ is a splitting field of $A$ then [25, Sect.5.4] implies 
that $\mathcal{M}(V)$ is unchanged under extension of the base field.

b) Small multiplicities. For the cases $[V: S]=1$ and $[V: S]=2$ we have precise descriptions of $\mathcal{L}_{S}(V)$ and $\mathcal{L}_{S}^{2}(V)$. To this end let $e \in A$ be a primitive idempotent such that $e A / \operatorname{rad}(e A) \cong S$. Then $V e \subseteq V$ is an $e A e$-module having $S e$ as its only constituent, and we have $[V e: S e]=[V: S]$ and $\operatorname{End}_{e A e}(S e) \cong$ $\operatorname{End}_{A}(S)$. By [25, Sect.5.4] we have $\mathcal{L}_{S}^{\infty}(V) \cong \mathcal{M}(V e)$ as lattices. Thus if $[V: S]=1$ then we have $\left|\mathcal{L}_{S}(V)\right|=1$ and $\mathcal{L}_{S}^{2}(V)=\emptyset$.

If $[V: S]=2$ then we have either $V e \cong S e \oplus S e$ or $V e \cong\left[\begin{array}{c}S e \\ S e\end{array}\right]$. In the latter case we have $\mathcal{L}_{S}^{2}(V)=\emptyset$ and $\mathcal{L}_{S}(V)=\left\{L^{\prime}, L\right\}$ where $L^{\prime}<L$. In the former case we have $\mathcal{L}_{S}^{2}(V)=\{U\}$ and $\mathcal{L}_{S}(V)=\left\{L_{i} ; i \in \mathcal{I}_{U}\right\}$, where we may identify $\mathcal{I}_{U}=\operatorname{End}_{A}(S) \dot{\cup}\{\infty\}=\operatorname{End}_{e A e}(S e) \dot{\cup}\{\infty\}=\mathcal{I}_{V e}$, thus $\left|\mathcal{L}_{S}(V)\right| \geq 3$. We have $L_{i}+L_{j}=U$ for all $i \neq j \in \mathcal{I}_{U}$, hence $\left\{L_{i}+\operatorname{rad}(U) ; i \in \mathcal{I}_{U}\right\}$ is in bijection with the maximal submodules of $U$, and $\mathcal{L}_{S}(V)$ is the unique dotted-line for $U$.

\section{The Young module $Y_{\mathbb{F}_{2}}^{[n-2,2]}$}

Let $p=2$ and $n \geq 5$ and $Y:=Y^{[n-2,2]}$. We determine the submodule structure of $Y_{\mathbb{F}_{2}}$, as is indicated in Table 1 .

(3.1) The cases $n \equiv \pm 1(\bmod 4)$. By $(2.6)$ and $(2.3)$ for $n \equiv 3(\bmod 4)$ we have $Y_{\mathbb{F}_{2}} \cong D^{[n-2,2]}$. For $n \equiv 1(\bmod 4)$ we have $Y_{\mathbb{F}_{2}} \sim 2 \cdot 1_{\mathbb{F}_{2}} \oplus D^{[n-2,2]}$. Since $Y_{\mathbb{F}_{2}}$ is indecomposable and self-dual, this determines the submodule structure.

(3.2) The case $n \equiv 2(\bmod 4)$. We have a Specht filtration $Y \cong\left[\frac{S_{\mathbb{Z}_{(2)}}^{[n-1,1]}}{S_{\mathbb{Z}_{(2)}}^{[n-2]}}\right]$, yielding a filtration with uniserial layers

$$
Y_{\mathbb{F}_{2}} \cong\left[\begin{array}{c}
D^{[n-1,1]} \\
1_{\mathbb{F}_{2}} \\
\hline D^{[n-2,2]} \\
1_{\mathbb{F}_{2}} \\
D^{[n-1,1]}
\end{array}\right] .
$$

Letting $\widehat{Y}:=Y^{[n-1,2]}$, we have $\left.\widehat{Y}\right|_{\mathcal{S}_{n}} \cong Y$, implying $\left.Y_{\mathbb{F}_{2}} \cong D^{[n-1,2]}\right|_{\mathcal{S}_{n}}$. Thus by (2.5) we have $\operatorname{soc}\left(Y_{\mathbb{F}_{2}}\right) \cong Y_{\mathbb{F}_{2}} / \operatorname{rad}\left(Y_{\mathbb{F}_{2}}\right) \cong D^{[n-1,1]}$. Since $\operatorname{rad}\left(Y_{\mathbb{F}_{2}}\right) / \operatorname{soc}\left(Y_{\mathbb{F}_{2}}\right)$ is self-dual having a submodule of shape $\left[\begin{array}{c}D^{[n-2,2]} \\ 1_{\mathbb{F}_{2}}\end{array}\right]$, we conclude that $Y_{\mathbb{F}_{2}} \cong$ $\left.D^{[n-1,2]}\right|_{\mathcal{S}_{n}}$ is uniserial, which also proves the assertion left open in (2.5). 
(3.3) The case $n \equiv 0(\bmod 4)$. We have a Specht filtration $Y \cong\left[\begin{array}{c}\frac{1_{\mathbb{Z}_{(2)}}}{S_{\mathbb{Z}_{(2)}}^{[n-1,1]}} \\ \hline S_{\mathbb{Z}_{(2)}}^{[n-2,2]}\end{array}\right]$, by (2.3) yielding a filtration with uniserial layers

$$
Y_{\mathbb{F}_{2}} \cong\left[\begin{array}{c}
\frac{1_{\mathbb{F}_{2}}}{D^{[n-1,1]}} \\
\frac{1_{\mathbb{F}_{2}}}{D^{[n-2,2]}} \\
D^{[n-1,1]}
\end{array}\right] .
$$

Hence from self-duality we deduce $1_{\mathbb{F}_{2}} \oplus D^{[n-1,1]} \leq \operatorname{soc}\left(Y_{\mathbb{F}_{2}}\right) \cong Y_{\mathbb{F}_{2}} / \operatorname{rad}\left(Y_{\mathbb{F}_{2}}\right)$, implying equality and $\operatorname{rad}\left(Y_{\mathbb{F}_{2}}\right) / \operatorname{soc}\left(Y_{\mathbb{F}_{2}}\right) \cong D^{[n-2,2]}$.

Let $L_{0}^{\prime}, L_{1}^{\prime}<Y_{\mathbb{F}_{2}}$ be the unique submodules isomorphic to $1_{\mathbb{F}_{2}}$ and $D^{[n-1,1]}$, respectively. Let $L_{2}<Y_{\mathbb{F}_{2}}$ be the unique $D^{[n-2,2]}$-local submodule, hence we have $L_{1}^{\prime}<L_{2}$ and $L_{2} \cong\left[\begin{array}{c}D^{[n-2,2]} \\ D^{[n-1,1]}\end{array}\right]$. There is a subquotient of shape $\left[\begin{array}{c}D^{[n-1,1]} \\ D^{[n-2,2]}\end{array}\right]$, hence there is a $D^{[n-1,1]}$-local submodule $L_{1}<Y_{\mathbb{F}_{2}}$ such that $L_{1}^{\prime}<L_{2}<L_{1}$, and thus $L_{1}^{\prime}$ and $L_{1}$ are the only $D^{[n-1,1]}$-local submodules.

Let $\widehat{L}_{1}<Y_{\mathbb{F}_{2}}$ be the unique submodule such that $Y_{\mathbb{F}_{2}} / \widehat{L}_{1} \cong D^{[n-1,1]}$. Thus $\widehat{L}_{1} / L_{1}^{\prime} \sim 2 \cdot 1_{\mathbb{F}_{2}} \oplus D^{[n-2,2]}$ is self-dual, and has a submodule isomorphic to $L_{2} / L_{1}^{\prime} \cong D^{[n-2,2]}$. Assume that $\widehat{L}_{1} / L_{1}^{\prime}$ is not semisimple, then $Y_{\mathbb{F}_{2}}$ has a subquotient of shape $\left[\begin{array}{l}1_{\mathbb{F}_{2}} \\ 1_{\mathbb{F}_{2}}\end{array}\right]$, which is an $\mathbb{F}_{2} C_{2}$-module for the natural epimorphic image $\mathcal{S}_{n} \rightarrow C_{2}$. Hence $\left.Y_{\mathbb{F}_{2}}\right|_{\mathcal{S}_{n-1}}$ also has a subquotient of this shape. But $\left.Y\right|_{\mathcal{S}_{n-1}} \cong 2 \cdot 1_{\mathbb{Z}_{(2)}} \oplus 2 \cdot Y^{[n-2,1]} \oplus Y^{[n-3,2]}$ implies $\left.Y_{\mathbb{F}_{2}}\right|_{\mathcal{S}_{n-1}} \cong 2 \cdot 1_{\mathbb{F}_{2}} \oplus 2 \cdot D^{[n-2,1]} \oplus$ $D^{[n-3,2]}$, a contradiction. Thus we have $\widehat{L}_{1} / L_{1}^{\prime} \cong 2 \cdot 1_{\mathbb{F}_{2}} \oplus D^{[n-2,2]}$.

Hence there is a $1_{\mathbb{F}_{2}}$-local submodule $L_{0}<Y_{\mathbb{F}_{2}}$ of shape $\left[\begin{array}{c}1_{\mathbb{F}_{2}} \\ D^{[n-1,1]}\end{array}\right]$, thus $\operatorname{rad}\left(L_{0}\right)=L_{1}^{\prime}$. Letting $V:=L_{0}^{\prime} \oplus L_{0}$ we get $\left(V / L_{1}^{\prime}\right) / \operatorname{rad}\left(V / L_{1}^{\prime}\right) \cong 1_{\mathbb{F}_{2}} \oplus 1_{\mathbb{F}_{2}}$, thus there are precisely three $1_{\mathbb{F}_{2}}$-local submodules $\left\{L_{0}^{\prime}, L_{0}, L_{0}^{\prime \prime}\right\}$, forming the unique dotted-line for $V$, where $L_{0}^{\prime \prime}$ has shape $\left[\begin{array}{c}1_{\mathbb{F}_{2}} \\ D^{[n-1,1]}\end{array}\right]$ and $\operatorname{rad}\left(L_{0}^{\prime \prime}\right)=L_{1}^{\prime}$.

It remains to determine $L_{1}$. Assume that $\operatorname{rad}\left(L_{1}\right)=L_{2}$, then we have $L_{1} \cap$ $L_{0}=L_{1} \cap L_{0}^{\prime \prime}=L_{1}^{\prime}$ and $L_{1} \cap L_{0}^{\prime}=\{0\}$, implying $Y_{\mathbb{F}_{2}} / L_{1} \cong 1_{\mathbb{F}_{2}} \oplus 1_{\mathbb{F}_{2}}$, a contradiction. Assume that $\operatorname{rad}\left(L_{1}\right)=L_{0}+L_{2}$ or $\operatorname{rad}\left(L_{1}\right)=L_{0}^{\prime \prime}+L_{2}$, then we have $Y_{\mathbb{F}_{2}}=L_{0}^{\prime} \oplus L_{1}$, again a contradiction. Hence we have $\operatorname{rad}\left(L_{1}\right)=L_{2}+L_{0}^{\prime}$.

\section{The Young module $Y_{\mathbb{F}_{2}}^{\left[n-2,1^{2}\right]}$}

Let $n \geq 5$ and $Y:=Y^{\left[n-2,1^{2}\right]}$. We determine the submodule structure of $Y_{\mathbb{F}_{2}}$, as is indicated in Table 2. To do so, in (4.1)-(4.4) we first consider the congruence 
classes of $n$ modulo 4 separately, which settles the case $n \equiv 2(\bmod 4)$, but only gives partial information for $n \not \equiv 2(\bmod 4)$. To complete the latter cases we in (4.5) set up an induction argument, which then is carried out in (4.6)-(4.8).

(4.1) The case $n \equiv 3(\bmod 4)$. By $(2.6)$ and $(2.3)$ we have a Specht filtration

$$
Y \cong\left[\begin{array}{c}
\frac{1_{\mathbb{Z}_{(2)}}}{S_{\mathbb{Z}_{(2)}^{[n-2,2]}}} \\
S_{\mathbb{Z}_{(2)}\left[n-2,1^{2}\right]}
\end{array}\right],
$$

implying $1_{\mathbb{F}_{2}} \oplus D^{[n-2,2]} \leq \operatorname{soc}\left(Y_{\mathbb{F}_{2}}\right) \cong Y_{\mathbb{F}_{2}} / \operatorname{rad}\left(Y_{\mathbb{F}_{2}}\right)$, and thus equality and $\operatorname{soc}\left(Y_{\mathbb{F}_{2}}\right)=\operatorname{rad}\left(Y_{\mathbb{F}_{2}}\right)$. Hence we have a filtration $Y_{\mathbb{F}_{2}} \cong\left[\frac{1_{\mathbb{F}_{2}} \oplus D^{[n-2,2]}}{1_{\mathbb{F}_{2}} \oplus D^{[n-2,2]}}\right]$.

Assume that $Y_{\mathbb{F}_{2}}$ has a submodule of shape $\left[\begin{array}{c}1_{\mathbb{F}_{2}} \\ 1_{\mathbb{F}_{2}}\end{array}\right]$, then it also has such a quotient module, and hence a submodule of shape $\left[\begin{array}{l}D^{[n-2,2]} \\ D^{[n-2,2]}\end{array}\right]$, contradicting its indecomposability. Similarly we deduce the non-existence of quotient modules of shape $\left[\begin{array}{c}1_{\mathbb{F}_{2}} \\ 1_{\mathbb{F}_{2}}\end{array}\right]$, and of submodules or quotient modules of shape $\left[\begin{array}{c}D^{[n-2,2]} \\ D^{[n-2,2]}\end{array}\right]$.

(4.2) The case $n \equiv 1(\bmod 4)$. We have a Specht filtration

$$
Y \cong\left[\frac{1_{\mathbb{Z}_{(2)}}}{\frac{S_{\mathbb{Z}_{(2)}}^{[n-2,2]}}{S_{\mathbb{Z}_{(2)}}^{\left[n-2,1^{2}\right]}}}\right] .
$$

Since $Y$ and $1_{\mathbb{Z}_{(2)}}$ are permutation lattices, and $\left[\begin{array}{c}1_{\mathbb{F}_{2}} \\ 1_{\mathbb{F}_{2}}\end{array}\right]$ is a permutation module lifting to the regular permutation lattice $\mathbb{Z}_{(2)} C_{2}$ for the natural epimorphic image $\mathcal{S}_{n} \rightarrow C_{2}$, we obtain $\operatorname{dim}_{\mathbb{F}_{2}}\left(\operatorname{Hom}_{\mathcal{S}_{n}}\left(1_{\mathbb{F}_{2}}, Y_{\mathbb{F}_{2}}\right)\right)=\operatorname{dim}_{\mathbb{Q}}\left(\operatorname{Hom}_{\mathcal{S}_{n}}\left(1_{\mathbb{Q}}, Y_{\mathbb{Q}}\right)\right)=$ 1 and $\operatorname{dim}_{\mathbb{F}_{2}}\left(\operatorname{Hom}_{\mathcal{S}_{n}}\left(\left[\begin{array}{c}1_{\mathbb{F}_{2}} \\ 1_{\mathbb{F}_{2}}\end{array}\right], Y_{\mathbb{F}_{2}}\right)\right)=\operatorname{dim}_{\mathbb{Q}}\left(\operatorname{Hom}_{\mathcal{S}_{n}}\left(1_{\mathbb{Q}} \oplus 1_{\mathbb{Q}}^{-}, Y_{\mathbb{Q}}\right)\right)=1$. Thus $Y_{\mathbb{F}_{2}}$ has a unique submodule isomorphic to $1_{\mathbb{F}_{2}}$, and no submodule of shape $\left[\begin{array}{c}1_{\mathbb{F}_{2}} \\ 1_{\mathbb{F}_{2}}\end{array}\right]$. Similarly, $Y_{\mathbb{F}_{2}}$ has a unique quotient module isomorphic to $1_{\mathbb{F}_{2}}$, and none of shape $\left[\begin{array}{c}1_{\mathbb{F}_{2}} \\ 1_{\mathbb{F}_{2}}\end{array}\right]$. Thus we have a filtration with uniserial layers

$$
Y_{\mathbb{F}_{2}} \cong\left[\begin{array}{c}
1_{\mathbb{F}_{2}} \\
D^{[n-2,2]} \\
1_{\mathbb{F}_{2}} \\
\hline 1_{\mathbb{F}_{2}} \\
D^{[n-2,2]} \\
1_{\mathbb{F}_{2}}
\end{array}\right]
$$


Let $S_{\mathbb{F}_{2}}^{\left[n-2,1^{2}\right]} \cong U<Y_{\mathbb{F}_{2}}$ be the submodule affording the above filtration, and let $W<Y_{\mathbb{F}_{2}}$ such that $U \not Z W$ and $W /(W \cap U) \cong(W+U) / U$ is simple. Hence we have $W /(W \cap U) \cong 1_{\mathbb{F}_{2}}$, and thus $W \cap U=\operatorname{rad}(U)$, implying $\operatorname{soc}^{2}\left(Y_{\mathbb{F}_{2}}\right)=$ $\operatorname{rad}(U) \cong\left[\begin{array}{c}D^{[n-2,2]} \\ 1_{\mathbb{F}_{2}}\end{array}\right]$. Hence we have $Y_{\mathbb{F}_{2}} / \operatorname{rad}^{2}\left(Y_{\mathbb{F}_{2}}\right) \cong\left[\begin{array}{c}1_{\mathbb{F}_{2}} \\ D^{[n-2,2]}\end{array}\right]$, implying $\operatorname{soc}^{2}\left(Y_{\mathbb{F}_{2}}\right)<\operatorname{rad}^{2}\left(Y_{\mathbb{F}_{2}}\right)$ and $\operatorname{rad}^{2}\left(Y_{\mathbb{F}_{2}}\right) / \operatorname{soc}^{2}\left(Y_{\mathbb{F}_{2}}\right) \sim 2 \cdot 1_{\mathbb{F}_{2}}$.

(4.3) The case $n \equiv 0(\bmod 4)$. We have a Specht filtration

$$
Y \cong\left[\begin{array}{l}
\frac{S_{\mathbb{Z}_{(2)}}^{n-1,1]}}{S_{\mathbb{Z}_{(2)}}^{[n-2,2]}} \\
S_{\mathbb{Z}_{(2)}}^{\left[n-2,1^{2}\right]}
\end{array}\right] .
$$

We determine $\operatorname{soc}\left(Y_{\mathbb{F}_{2}}\right)$. From $M_{\mathbb{F}_{2}}^{\left[n-2,1^{2}\right]} \cong Y_{\mathbb{F}_{2}}^{[n-1,1]} \oplus Y_{\mathbb{F}_{2}}$ we deduce that $1_{\mathbb{F}_{2}}$ is not a submodule of $Y_{\mathbb{F}_{2}}$. By (2.5) from $\left.D^{[n-1,1]}\right|_{\mathcal{S}_{n-2}} \cong\left[\begin{array}{c}1_{\mathbb{F}_{2}} \\ D^{[n-3,1]} \\ 1_{\mathbb{F}_{2}}\end{array}\right]$ we get $\operatorname{dim}_{\mathbb{F}_{2}}\left(\operatorname{Hom}_{\mathcal{S}_{n}}\left(D^{[n-1,1]}, M_{\mathbb{F}_{2}}^{\left[n-2,1^{2}\right]}\right)\right)=\operatorname{dim}_{\mathbb{F}_{2}}\left(\operatorname{Hom}_{\mathcal{S}_{n-2}}\left(\left.D^{[n-1,1]}\right|_{\mathcal{S}_{n-2}}, 1_{\mathbb{F}_{2}}\right)\right)=1$, hence there is a unique submodule of $Y_{\mathbb{F}_{2}}$ isomorphic to $D^{[n-1,1]}$. As $1_{\mathbb{F}_{2}}$ is not a quotient module of $\left.D^{[n-2,2]}\right|_{\mathcal{S}_{n-2}}$, we get $\operatorname{dim}_{\mathbb{F}_{2}}\left(\operatorname{Hom}_{\mathcal{S}_{n}}\left(D^{[n-2,2]}, M_{\mathbb{F}_{2}}^{\left[n-2,1^{2}\right]}\right)\right)=$ $\operatorname{dim}_{\mathbb{F}_{2}}\left(\operatorname{Hom}_{\mathcal{S}_{n-2}}\left(\left.D^{[n-2,2]}\right|_{\mathcal{S}_{n-2}}, 1_{\mathbb{F}_{2}}\right)\right)=0$, hence $D^{[n-2,2]}$ is not a submodule of $Y_{\mathbb{F}_{2}}$. Thus we have $\operatorname{soc}\left(Y_{\mathbb{F}_{2}}\right) \cong Y_{\mathbb{F}_{2}} / \operatorname{rad}\left(Y_{\mathbb{F}_{2}}\right) \cong D^{[n-1,1]}$.

Letting $\widehat{Y}:=Y^{\left[n-1,1^{2}\right]}$, we get $\left.\widehat{Y}\right|_{\mathcal{S}_{n}} \cong Y^{[n-1,1]} \oplus Y$ where $Y_{\mathbb{F}_{2}}^{[n-1,1]} \cong M_{\mathbb{F}_{2}}^{[n-1,1]} \cong$ $\left[\begin{array}{c}1_{\mathbb{F}_{2}} \\ D^{[n-1,1]} \\ 1_{\mathbb{F}_{2}}\end{array}\right]$. By (4.2) we have $\operatorname{soc}\left(\widehat{Y}_{\mathbb{F}_{2}}\right) \cong 1_{\mathbb{F}_{2}}$, hence factoring it out yields $\left.\left(\widehat{Y}_{\mathbb{F}_{2}} / \operatorname{soc}\left(\widehat{Y}_{\mathbb{F}_{2}}\right)\right)\right|_{\mathcal{S}_{n}} \cong\left[\begin{array}{c}1_{\mathbb{F}_{2}} \\ D^{[n-1,1]}\end{array}\right] \oplus Y_{\mathbb{F}_{2}}$. We have $\left.\left(\operatorname{soc}^{2}\left(\widehat{Y}_{\mathbb{F}_{2}}\right) / \operatorname{soc}\left(\widehat{Y}_{\mathbb{F}_{2}}\right)\right)\right|_{\mathcal{S}_{n}} \cong$ $\left.D^{[n-1,2]}\right|_{\mathcal{S}_{n}} \cong\left[\begin{array}{c}D^{[n-1,1]} \\ D^{[n-2,2]} \\ D^{[n-1,1]}\end{array}\right]$. Thus projecting $D^{[n-1,2]} \leq \widehat{Y}_{\mathbb{F}_{2}} / \operatorname{soc}\left(\widehat{Y}_{\mathbb{F}_{2}}\right)$ into the direct summand $Y_{\mathbb{F}_{2}}$ of $\left.\left(\widehat{Y}_{\mathbb{F}_{2}} / \operatorname{soc}\left(\widehat{Y}_{\mathbb{F}_{2}}\right)\right)\right|_{\mathcal{S}_{n}}$, we obtain a submodule of $Y_{\mathbb{F}_{2}}$ of Loewy length 3 , which hence is isomorphic to $\left.D^{[n-1,2]}\right|_{\mathcal{S}_{n}}$.

Let $V:=\operatorname{rad}\left(Y_{\mathbb{F}_{2}}\right) / \operatorname{soc}\left(Y_{\mathbb{F}_{2}}\right)$. Hence $V \sim 2 \cdot 1_{\mathbb{F}_{2}} \oplus D^{[n-1,1]} \oplus 2 \cdot D^{[n-2,2]}$ is self-dual, and has a filtration with uniserial second layer

$$
V \cong\left[\begin{array}{c}
\frac{1_{\mathbb{F}_{2}}}{D^{[n-2,2]}} \\
\frac{D^{[n-1,1]}}{1_{\mathbb{F}_{2}} \oplus D^{[n-2,2]}}
\end{array}\right]
$$


Let $L_{1}<V$ be the unique $D^{[n-1,1]}$-local submodule. From having a submodule of shape $\left[\begin{array}{l}D^{[n-1,1]} \\ D^{[n-2,2]}\end{array}\right]$ and being self-dual we deduce that there are $D^{[n-2,2]}$ local submodules $L_{2}, L_{2}^{\prime}<V$ such that $L_{2}^{\prime}<L_{1}<L_{2}$. Thus $L_{2}$ and $L_{2}^{\prime}$ are the only $D^{[n-2,2]}$-local submodules, $L_{2}^{\prime}$ is the unique submodule isomorphic to $D^{[n-2,2]}$, and we have $L_{1} \cong\left[\begin{array}{c}D^{[n-1,1]} \\ D^{[n-2,2]}\end{array}\right]$. In particular this implies that $V$ has no submodule isomorphic to $D^{[n-1,1]}$.

We have $\left.Y\right|_{\mathcal{S}_{n-1}} \cong 3 \cdot Y^{[n-2,1]} \oplus Y^{\left[n-3,1^{2}\right]}$, where $Y_{\mathbb{F}_{2}}^{[n-2,1]} \cong D^{[n-2,1]}$ does not belong to the principal 2-block of $\mathcal{S}_{n-1}$. Thus $\left.V\right|_{\mathcal{S}_{n-1}} \cong D^{[n-2,1]} \oplus Y_{\mathbb{F}_{2}}^{\left[n-3,1^{2}\right]}$, implying that $V$ is indecomposable. Moreover, by (4.1) there is a unique submodule $L_{0}^{\prime}<V$ isomorphic to $1_{\mathbb{F}_{2}}$, hence we have $\operatorname{soc}(V)=L_{0}^{\prime} \oplus L_{2}^{\prime} \cong 1_{\mathbb{F}_{2}} \oplus D^{[n-2,2]}$.

We determine the structure of $L_{2}$. Assume that $\operatorname{rad}\left(L_{2}\right)=L_{1}$, then $V / L_{2} \sim$ $2 \cdot 1_{\mathbb{F}_{2}}$, and projecting into the direct summand $Y_{\mathbb{F}_{2}}^{\left[n-3,1^{2}\right]}$ of $\left.V\right|_{\mathcal{S}_{n-1}}$ we obtain a quotient module $\sim 2 \cdot 1_{\mathbb{F}_{2}}$, contradicting (4.1). Hence we have $L_{2} \sim 1_{\mathbb{F}_{2}} \oplus$ $D^{[n-1,1]} \oplus 2 \cdot D^{[n-2,2]}$. Assume that $L_{2} \cap \operatorname{soc}(V)=L_{2}^{\prime}$, then we have $V \cong L_{0}^{\prime} \oplus L_{2}$, a contradiction. Hence $\operatorname{soc}(V)=L_{0}^{\prime} \oplus L_{2}^{\prime}<L_{2}$ and thus $\operatorname{rad}\left(L_{2}\right)=L_{0}^{\prime} \oplus L_{1}$.

(4.4) The case $n \equiv 2(\bmod 4)$. We have a Specht filtration

$$
Y \cong\left[\begin{array}{l}
\frac{S_{\mathbb{Z}_{(2)}}^{[n-1,1]}}{S_{\mathbb{Z}_{(2)}}^{[n-2,2]}} \\
\hline S_{\mathbb{Z}_{(2)}}^{\left[n-2,1^{2}\right]}
\end{array}\right],
$$

and proceeding as in (4.3) we again find $\operatorname{soc}\left(Y_{\mathbb{F}_{2}}\right) \cong Y_{\mathbb{F}_{2}} / \operatorname{rad}\left(Y_{\mathbb{F}_{2}}\right) \cong D^{[n-1,1]}$.

Letting $\widehat{Y}:=Y^{\left[n-1,1^{2}\right]}$, we have $\left.\widehat{Y}\right|_{\mathcal{S}_{n}} \cong Y^{[n-1,1]} \oplus Y$. By (4.1) we have $\operatorname{soc}\left(\widehat{Y}_{\mathbb{F}_{2}}\right) \cong 1_{\mathbb{F}_{2}} \oplus D^{[n-1,2]}$, thus factoring out the submodule $L<\widehat{Y}_{\mathbb{F}_{2}}$ isomorphic to $1_{\mathbb{F}_{2}}$ yields $\left.\left(\widehat{Y}_{\mathbb{F}_{2}} / L\right)\right|_{\mathcal{S}_{n}} \cong\left[\begin{array}{c}1_{\mathbb{F}_{2}} \\ D^{[n-1,1]}\end{array}\right] \oplus Y_{\mathbb{F}_{2}}$, which by (3.2) has a submodule

$$
\left.D^{[n-1,2]}\right|_{\mathcal{S}_{n}} \cong\left[\begin{array}{c}
D^{[n-1,1]} \\
1_{\mathbb{F}_{2}} \\
D^{[n-2,2]} \\
1_{\mathbb{F}_{2}} \\
D^{[n-1,1]}
\end{array}\right] \text {. }
$$

Thus projecting the submodule $D^{[n-1,2]} \leq \widehat{Y}_{\mathbb{F}_{2}} / L$ into the direct summand $Y_{\mathbb{F}_{2}}$ of $\left.\left(\widehat{Y}_{\mathbb{F}_{2}} / L\right)\right|_{\mathcal{S}_{n}}$, we obtain a submodule of $Y_{\mathbb{F}_{2}}$ of Loewy length 5 , which hence is isomorphic to $\left.D^{[n-1,2]}\right|_{\mathcal{S}_{n}}$.

Let $V:=\operatorname{rad}\left(Y_{\mathbb{F}_{2}}\right) / \operatorname{soc}\left(Y_{\mathbb{F}_{2}}\right)$. Hence $V \sim 4 \cdot 1_{\mathbb{F}_{2}} \oplus D^{[n-1,1]} \oplus 2 \cdot D^{[n-2,2]}$ is 
self-dual, and its unique $D^{[n-1,1]}$-local submodule is given as

$$
L_{1} \cong\left[\begin{array}{c}
D^{[n-1,1]} \\
1_{\mathbb{F}_{2}} \\
D^{[n-2,2]} \\
1_{\mathbb{F}_{2}}
\end{array}\right]
$$

Hence by self-duality there are $D^{[n-2,2]}$-local submodules $L_{2}, L_{2}^{\prime} \leq V$ such that $L_{2}^{\prime}<L_{1}<L_{2}$. Thus $L_{2}$ and $L_{2}^{\prime} \cong\left[\begin{array}{c}D^{[n-2,2]} \\ 1_{\mathbb{F}_{2}}\end{array}\right]$ are the only $D^{[n-2,2]}$-local submodules of $V$.

We have $\left.Y\right|_{\mathcal{S}_{n-1}} \cong 3 \cdot Y^{[n-2,1]} \oplus Y^{\left[n-3,1^{2}\right]}$, where $Y_{\mathbb{F}_{2}}^{[n-2,1]} \cong D^{[n-2,1]}$ does not belong to the principal 2-block of $\mathcal{S}_{n-1}$. Thus we have $\left.V\right|_{\mathcal{S}_{n-1}} \cong D^{[n-2,1]} \oplus$ $Y_{\mathbb{F}_{2}}^{\left[n-3,1^{2}\right]}$, and (4.2) implies that $\operatorname{soc}^{2}(V)=L_{2}^{\prime}$. Hence we have $V / \operatorname{rad}^{2}(V) \cong$ $\left[\begin{array}{c}1_{\mathbb{F}_{2}} \\ D^{[n-2,2]}\end{array}\right]$ and $\operatorname{soc}^{2}(V)<\operatorname{rad}^{2}(V)$, where $\operatorname{rad}^{2}(V) / \operatorname{soc}^{2}(V) \sim 2 \cdot 1_{\mathbb{F}_{2}} \oplus D^{[n-1,1]}$ is self-dual and has a submodule isomorphic to $L_{1} / L_{2}^{\prime} \cong\left[\begin{array}{c}D^{[n-1,1]} \\ 1_{\mathbb{F}_{2}}\end{array}\right]$. Hence $\operatorname{rad}^{2}(V) / \operatorname{soc}^{2}(V)$ is uniserial, implying that $V$ is uniserial as well, completing the case $n \equiv 2(\bmod 4)$.

(4.5) The cases $n \not \equiv 2(\bmod 4)$, continued. To complete the cases $n \not \equiv 2$ $(\bmod 4)$, where $n \geq 5$, we proceed by induction. For $n=5$ we explicitly confirm that $Y_{\mathbb{F}_{2}}^{\left[3,1^{2}\right]}$ is uniserial as described in (1.5). Hence we now let $n \geq 6$.

(4.6) The case $n \equiv 0(\bmod 4)$, continued. We keep the notation of (4.3). Assume that there is an $1_{\mathbb{F}_{2}}$-local submodule $L_{0}<V$ such that $L_{0}^{\prime} \not \leq L_{0}$, then $V$ has a subquotient isomorphic to $1_{\mathbb{F}_{2}} \oplus 1_{\mathbb{F}_{2}}$. Since $\left.V\right|_{\mathcal{S}_{n-1}} \cong D^{[n-2,1]} \oplus Y_{\mathbb{F}_{2}}^{\left[n-3,1^{2}\right]}$ this implies that $Y_{\mathbb{F}_{2}}^{\left[n-3,1^{2}\right]}$ has a subquotient isomorphic to $1_{\mathbb{F}_{2}} \oplus 1_{\mathbb{F}_{2}}$, by induction contradicting (4.8). Hence there is an $1_{\mathbb{F}_{2}}$-local submodule $L_{0}^{\prime}<L_{0}<V$, thus $L_{0}^{\prime}$ and $L_{0}$ are the only $1_{\mathbb{F}_{2}}$-local submodules of $V$.

Since we have $L_{0} \not \leq L_{2}$ and $L_{0}<V$, we conclude that $\operatorname{rad}\left(L_{0}\right)=L_{0} \cap L_{2}$ and $\operatorname{rad}\left(L_{0}\right) \in\left\{L_{0}^{\prime}, L_{0}^{\prime} \oplus L_{2}^{\prime}, L_{0}^{\prime} \oplus L_{1}\right\}$. Assume that $\operatorname{rad}\left(L_{0}\right)=L_{0}^{\prime}$, then $Y_{\mathbb{F}_{2}}^{\left[n-3,1^{2}\right]}$ has a submodule $\sim 2 \cdot 1_{\mathbb{F}_{2}}$, contradicting (4.1). Assume that $\operatorname{rad}\left(L_{0}\right)=L_{0}^{\prime} \oplus L_{1}=$ $\operatorname{rad}\left(L_{2}\right)$, then we have $V / L_{0} \cong D^{[n-2,2]}$, thus by self-duality there is $W<L_{0}$ such that $V / W \cong\left[\begin{array}{c}D^{[n-2,2]} \\ D^{[n-1,1]}\end{array}\right]$, contradicting $L_{0} / \operatorname{rad}\left(L_{0}\right) \cong 1_{\mathbb{F}_{2}}$. Hence we have $\operatorname{rad}\left(L_{0}\right)=L_{0}^{\prime} \oplus L_{2}^{\prime}$, completing the case $n \equiv 0(\bmod 4)$.

(4.7) The case $n \equiv 1(\bmod 4)$, continued. We keep the notation of $(4.2)$. We have $\left.Y\right|_{\mathcal{S}_{n-1}} \cong Y^{[n-2,1]} \oplus Y^{\left[n-3,1^{2}\right]}$, where by (4.3) $Y_{\mathbb{F}_{2}}^{\left[n-3,1^{2}\right]} / \operatorname{rad}\left(Y_{\mathbb{F}_{2}}^{\left[n-3,1^{2}\right]}\right) \cong$ 
$\operatorname{soc}\left(Y_{\mathbb{F}_{2}}^{\left[n-3,1^{2}\right]}\right) \cong D^{[n-2,1]}$ and $Y_{\mathbb{F}_{2}}^{[n-2,1]} \cong M_{\mathbb{F}_{2}}^{[n-2,1]} \cong\left[\begin{array}{c}1_{\mathbb{F}_{2}} \\ D^{[n-2,1]} \\ 1_{\mathbb{F}_{2}}\end{array}\right]$.

Letting $V:=\operatorname{rad}\left(Y_{\mathbb{F}_{2}}\right) / \operatorname{soc}\left(Y_{\mathbb{F}_{2}}\right)$, where $\operatorname{soc}\left(Y_{\mathbb{F}_{2}}\right) \cong Y_{\mathbb{F}_{2}} / \operatorname{rad}\left(Y_{\mathbb{F}_{2}}\right) \cong 1_{\mathbb{F}_{2}}$, yields $\left.V\right|_{\mathcal{S}_{n-1}} \cong D^{[n-2,1]} \oplus Y_{\mathbb{F}_{2}}^{\left[n-3,1^{2}\right]}$. Assume that $\operatorname{rad}(V) / \operatorname{soc}(V) \cong 1_{\mathbb{F}_{2}} \oplus 1_{\mathbb{F}_{2}}$, then $Y_{\mathbb{F}_{2}}^{\left[n-3,1^{2}\right]}$ has a subquotient isomorphic to $1_{\mathbb{F}_{2}} \oplus 1_{\mathbb{F}_{2}}$, by induction contradicting (4.6). Hence $\operatorname{rad}(V) / \operatorname{soc}(V) \cong\left[\begin{array}{l}1_{\mathbb{F}_{2}} \\ 1_{\mathbb{F}_{2}}\end{array}\right]$, completing the case $n \equiv 1(\bmod 4)$.

(4.8) The case $n \equiv 3(\bmod 4)$, continued. We keep the notation of (4.1). We have $\left.Y\right|_{\mathcal{S}_{n-1}} \cong Y^{[n-2,1]} \oplus Y^{\left[n-3,1^{2}\right]}$, hence letting $M:=M_{\mathbb{Z}_{(2)}^{[n-2,1]} \cong Y^{[n-2,1]}}^{[n}$ and $Y^{\prime}:=Y^{\left[n-3,1^{2}\right]}$ we by (4.4) have

$$
\left.Y_{\mathbb{F}_{2}}\right|_{\mathcal{S}_{n-1}} \cong M_{\mathbb{F}_{2}} \oplus Y_{\mathbb{F}_{2}}^{\prime} \cong\left[\begin{array}{c}
1_{\mathbb{F}_{2}} \\
D^{[n-2,1]} \\
1_{\mathbb{F}_{2}}
\end{array}\right] \oplus\left[\begin{array}{c}
D^{[n-2,1]} \\
1_{\mathbb{F}_{2}} \\
D^{[n-3,2]} \\
1_{\mathbb{F}_{2}} \\
D^{[n-2,1]} \\
1_{\mathbb{F}_{2}} \\
D^{[n-3,2]} \\
1_{\mathbb{F}_{2}} \\
D^{[n-2,1]}
\end{array}\right] .
$$

Let $L_{0}^{\prime}, L_{2}^{\prime}<Y_{\mathbb{F}_{2}}$ be the unique submodules isomorphic to $1_{\mathbb{F}_{2}}$ and $D^{[n-2,2]}$, respectively. Assume that there is a $D^{[n-2,2]}$-local submodule $L_{2}<Y_{\mathbb{F}_{2}}$ such that $L_{2}^{\prime} \not \leq L_{2}$, then $Y_{\mathbb{F}_{2}}$ has a subquotient isomorphic to $D^{[n-2,2]} \oplus D^{[n-2,2]}$. Thus we conclude that $\left.Y_{\mathbb{F}_{2}}\right|_{\mathcal{S}_{n-1}}$ has a subquotient isomorphic to $D^{[n-3,2]} \oplus$ $D^{[n-3,2]}$, a contradiction. Hence we have $L_{2}^{\prime}<L_{2}<Y_{\mathbb{F}_{2}}$, thus $L_{2}^{\prime}$ and $L_{2}$ are the only $D^{[n-2,2]}$-local submodules, and since $L_{2}$ has Loewy length 2 and $\operatorname{rad}\left(L_{2}\right)>L_{2}^{\prime}$ we conclude that $\operatorname{rad}\left(L_{2}\right)=\operatorname{soc}\left(Y_{\mathbb{F}_{2}}\right)=L_{0}^{\prime} \oplus L_{2}^{\prime}$.

(*) Assume that there is a $1_{\mathbb{F}_{2}}$-local submodule $L_{0}<Y_{\mathbb{F}_{2}}$ such that $L_{0}^{\prime} \not \leq L_{0}$, then we have $L_{2}^{\prime}<L_{0}$ and $L_{0} \cong\left[\begin{array}{c}1_{\mathbb{F}_{2}} \\ D^{[n-2,2]}\end{array}\right]$. To proceed towards a contradiction, let $\widehat{L}_{2}<Y_{\mathbb{F}_{2}}$ be the unique submodule such that $Y_{\mathbb{F}_{2}} / \widehat{L}_{2} \cong D^{[n-2,2]}$, hence we have $\widehat{L}_{2} / L_{2}^{\prime} \cong 1_{\mathbb{F}_{2}} \oplus 1_{\mathbb{F}_{2}}$. Moreover, let $\alpha \in \operatorname{End}_{\mathcal{S}_{n}}\left(Y_{\mathbb{F}_{2}}\right)$ such that $\operatorname{im}(\alpha)=L_{2}^{\prime} \cong$ $D^{[n-2,2]}$, thus we have $\alpha^{2}=0$ and $\operatorname{ker}(\alpha)=\widehat{L}_{2}$.

Since $M$ is a permutation lattice and $Y^{\prime}$ is a direct summand of a permutation lattice we get $\operatorname{dim}_{\mathbb{F}_{2}}\left(\operatorname{End}_{\mathcal{S}_{n-1}}\left(M_{\mathbb{F}_{2}}\right)\right)=2$ and $\operatorname{dim}_{\mathbb{F}_{2}}\left(\operatorname{End}_{\mathcal{S}_{n-1}}\left(Y_{\mathbb{F}_{2}}^{\prime}\right)\right)=3$. Hence there is an $\mathbb{F}_{2}$-basis $\left\{\operatorname{id}_{M}, \mu\right\}$ of $\operatorname{End}_{\mathcal{S}_{n-1}}\left(M_{\mathbb{F}_{2}}\right)$, where $\operatorname{im}(\mu)=\operatorname{soc}\left(M_{\mathbb{F}_{2}}\right) \cong 1_{\mathbb{F}_{2}}$ and $\mu^{2}=0$, and there is an $\mathbb{F}_{2}$-basis $\left\{\operatorname{id}_{Y^{\prime}}, \delta, \delta^{2}\right\}$ of $\operatorname{End}_{\mathcal{S}_{n-1}}\left(Y_{\mathbb{F}_{2}}^{\prime}\right)$, where $\operatorname{im}(\delta)=$ $\operatorname{rad}^{4}\left(Y_{\mathbb{F}_{2}}^{\prime}\right)=\operatorname{soc}^{5}\left(Y_{\mathbb{F}_{2}}^{\prime}\right)$ and hence $\operatorname{im}\left(\delta^{2}\right)=\operatorname{soc}\left(Y_{\mathbb{F}_{2}}^{\prime}\right) \cong D^{[n-2,1]}$ and $\delta^{3}=0$. Similarly, $\operatorname{dim}_{\mathbb{F}_{2}}\left(\operatorname{Hom}_{\mathcal{S}_{n-1}}\left(M_{\mathbb{F}_{2}}, Y_{\mathbb{F}_{2}}^{\prime}\right)\right)=\operatorname{dim}_{\mathbb{F}_{2}}\left(\operatorname{Hom}_{\mathcal{S}_{n-1}}\left(Y_{\mathbb{F}_{2}}^{\prime}, M_{\mathbb{F}_{2}}\right)\right)=1$, hence 
there are $0 \neq \rho \in \operatorname{Hom}_{\mathcal{S}_{n-1}}\left(M_{\mathbb{F}_{2}}, Y_{\mathbb{F}_{2}}^{\prime}\right)$ and $0 \neq \lambda \in \operatorname{Hom}_{\mathcal{S}_{n-1}}\left(Y_{\mathbb{F}_{2}}^{\prime}, M_{\mathbb{F}_{2}}\right)$ such that $\operatorname{im}(\rho)=\operatorname{soc}^{2}\left(Y_{\mathbb{F}_{2}}^{\prime}\right) \cong\left[\begin{array}{c}1_{\mathbb{F}_{2}} \\ D^{[n-2,1]}\end{array}\right]$ as well as $\operatorname{im}(\lambda)=\operatorname{soc}^{2}\left(M_{\mathbb{F}_{2}}\right) \cong\left[\begin{array}{c}D^{[n-2,1]} \\ 1_{\mathbb{F}_{2}}\end{array}\right]$. Thus $\operatorname{End}_{\mathcal{S}_{n-1}}\left(M_{\mathbb{F}_{2}} \oplus Y_{\mathbb{F}_{2}}^{\prime}\right)$ has $\left\{\operatorname{id}_{M}, \mu, \rho, \lambda, \operatorname{id}_{Y^{\prime}}, \delta, \delta^{2}\right\}$ as an $\mathbb{F}_{2}$-basis, whose multiplication table is given in Table 10; note that endomorphisms also act from the right.

Since by (3.2) $\left.D^{[n-2,2]}\right|_{\mathcal{S}_{n-1}}$ has Loewy length 5 such that $\operatorname{soc}\left(\left.D^{[n-2,2]}\right|_{\mathcal{S}_{n-1}}\right) \cong$ $D^{[n-2,1]}$, we conclude that $\alpha \in \operatorname{End}_{\mathcal{S}_{n-1}}\left(M_{\mathbb{F}_{2}} \oplus Y_{\mathbb{F}_{2}}^{\prime}\right)$ can be written as an $\mathbb{F}_{2^{-}}$ linear combination $\alpha=\delta+a \rho+b \lambda$, where $a, b \in \mathbb{F}_{2}$ and where we replace $\delta$ by $\delta+\delta^{2}$ if necessary. From $\alpha^{2}=(1+a b) \delta^{2}$ we deduce $a=b=1$. Hence we have $L_{2}^{\prime}=\operatorname{im}(\delta+\rho+\lambda)$ and $\widehat{L}_{2}=\operatorname{ker}(\delta+\rho+\lambda)$.

Thus we have $\operatorname{soc}^{3}\left(Y_{\mathbb{F}_{2}}^{\prime}\right)=\operatorname{soc}^{7}\left(Y_{\mathbb{F}_{2}}^{\prime}\right)^{\delta+\rho+\lambda}<L_{2}^{\prime}$, and since both induced maps $\lambda: Y_{\mathbb{F}_{2}}^{\prime} / \operatorname{soc}^{7}\left(Y_{\mathbb{F}_{2}}^{\prime}\right) \rightarrow \operatorname{soc}^{2}\left(M_{\mathbb{F}_{2}}\right)$ and $\delta: Y_{\mathbb{F}_{2}}^{\prime} / \operatorname{soc}^{7}\left(Y_{\mathbb{F}_{2}}^{\prime}\right) \rightarrow \operatorname{soc}^{5}\left(Y_{\mathbb{F}_{2}}^{\prime}\right) / \operatorname{soc}^{3}\left(Y_{\mathbb{F}_{2}}^{\prime}\right)$ are isomorphisms, we conclude that $L_{2}^{\prime} \cap M_{\mathbb{F}_{2}}=\{0\}$ and $L_{2}^{\prime} \cap Y_{\mathbb{F}_{2}}^{\prime}=\operatorname{soc}^{3}\left(Y_{\mathbb{F}_{2}}^{\prime}\right)$. Moreover, for $[m, y] \in \operatorname{ker}(\delta+\rho+\lambda)<M_{\mathbb{F}_{2}} \oplus Y_{\mathbb{F}_{2}}^{\prime}$ we have $\left[y^{\lambda}, y^{\delta}+m^{\rho}\right]=0$, hence $y^{\delta}=m^{\rho} \in \operatorname{soc}^{2}\left(Y_{\mathbb{F}_{2}}^{\prime}\right)$, implying $y \in \operatorname{soc}^{6}\left(Y_{\mathbb{F}_{2}}^{\prime}\right)<\operatorname{soc}^{7}\left(Y_{\mathbb{F}_{2}}^{\prime}\right)=\operatorname{ker}(\lambda)$. Thus we have $\widehat{L}_{2}<M_{\mathbb{F}_{2}} \oplus \operatorname{soc}^{6}\left(Y_{\mathbb{F}_{2}}^{\prime}\right)$.

Let $M_{\mathbb{F}_{2}}^{\prime}:=\operatorname{soc}^{6}\left(Y_{\mathbb{F}_{2}}^{\prime}\right) / \operatorname{soc}^{3}\left(Y_{\mathbb{F}_{2}}^{\prime}\right)$. Hence $M_{\mathbb{F}_{2}}$ and $M_{\mathbb{F}_{2}}^{\prime}$ have shape $\left[\begin{array}{c}1_{\mathbb{F}_{2}} \\ D^{[n-2,1]} \\ 1_{\mathbb{F}_{2}}\end{array}\right]$. Let $W:=\left(\left.L_{2}^{\prime}\right|_{\mathcal{S}_{n-1}}\right) / \operatorname{soc}^{3}\left(Y_{\mathbb{F}_{2}}^{\prime}\right)<M_{\mathbb{F}_{2}} \oplus M_{\mathbb{F}_{2}}^{\prime}$ and $V:=\left(\left.\widehat{L}_{2}\right|_{\mathcal{S}_{n-1}}\right) / \operatorname{soc}^{3}\left(Y_{\mathbb{F}_{2}}^{\prime}\right)<$ $M_{\mathbb{F}_{2}} \oplus M_{\mathbb{F}_{2}}^{\prime}$. Hence we deduce $\left(M_{\mathbb{F}_{2}} \oplus M_{\mathbb{F}_{2}}^{\prime}\right) / V \sim 1_{\mathbb{F}_{2}} \oplus D^{[n-2,1]}$ and $W \cong$ $\left[\begin{array}{c}D^{[n-2,1]} \\ 1_{\mathbb{F}_{2}}\end{array}\right]$, where $W \cap M_{\mathbb{F}_{2}}=W \cap M_{\mathbb{F}_{2}}^{\prime}=\{0\}$. Moreover, we have $W<V$ such that $V / W \cong 1_{\mathbb{F}_{2}} \oplus 1_{\mathbb{F}_{2}}$, hence there are submodules $U, U^{\prime}<V$ such that $U \cap U^{\prime}=W$ and $U+U^{\prime}=V$. Assume that both $U, U^{\prime} \leq \operatorname{soc}^{2}\left(M_{\mathbb{F}_{2}} \oplus M_{\mathbb{F}_{2}}^{\prime}\right)$, then $V \leq \operatorname{soc}^{2}\left(M_{\mathbb{F}_{2}} \oplus M_{\mathbb{F}_{2}}^{\prime}\right)$ as well. Since $V \sim 3 \cdot 1_{\mathbb{F}_{2}} \oplus D^{[n-2,1]}$ and $\operatorname{soc}^{2}\left(M_{\mathbb{F}_{2}} \oplus\right.$ $\left.M_{\mathbb{F}_{2}}^{\prime}\right) \sim 2 \cdot 1_{\mathbb{F}_{2}} \oplus 2 \cdot D^{[n-2,1]}$ this is a contradiction. Hence we may assume that $U \stackrel{\leq}{\not} \operatorname{soc}^{2}\left(M_{\mathbb{F}_{2}} \oplus M_{\mathbb{F}_{2}}^{\prime}\right)$, thus since $U / W \cong 1_{\mathbb{F}_{2}}$ we conclude that $U$ also has shape $\left[\begin{array}{c}1_{\mathbb{F}_{2}} \\ D^{[n-2,1]} \\ 1_{\mathbb{F}_{2}}\end{array}\right]$. Hence $U \cap M_{\mathbb{F}_{2}}=U \cap M_{\mathbb{F}_{2}}^{\prime}=\{0\}$, implying $M_{\mathbb{F}_{2}} \cong U \cong M_{\mathbb{F}_{2}}^{\prime}$.

Now $\left.Y^{[n-2,1]}\right|_{\mathcal{S}_{n-2}} \cong 2 \cdot Y^{[n-2]} \oplus Y^{[n-3,1]}$ implies $\left.\left.M_{\mathbb{F}_{2}}\right|_{\mathcal{S}_{n-2}} \cong Y_{\mathbb{F}_{2}}^{[n-2,1]}\right|_{\mathcal{S}_{n-2}} \cong$ $2 \cdot 1_{\mathbb{F}_{2}} \oplus D^{[n-3,1]}$. Moreover, we have $\left.Y^{\left[n-3,1^{2}\right]}\right|_{\mathcal{S}_{n-2}} \cong 3 \cdot Y^{[n-3,1]} \oplus Y^{\left[n-4,1^{2}\right]}$, hence $\left.Y_{\mathbb{F}_{2}}^{\prime}\right|_{\mathcal{S}_{n-2}} \cong 3 \cdot D^{[n-3,1]} \oplus Y_{\mathbb{F}_{2}}^{\left[n-4,1^{2}\right]}$, where by induction and (4.7) we have

$$
Y_{\mathbb{F}_{2}}^{\left[n-4,1^{2}\right]} \cong\left[\begin{array}{c}
1_{\mathbb{F}_{2}} \\
D^{[n-4,2]} \\
1_{\mathbb{F}_{2}} \\
1_{\mathbb{F}_{2}} \\
D^{[n-4,2]} \\
1_{\mathbb{F}_{2}}
\end{array}\right]
$$


Table 10: Multiplication table of $\operatorname{End}_{\mathcal{S}_{n-1}}\left(M_{\mathbb{F}_{2}} \oplus Y_{\mathbb{F}_{2}}^{\prime}\right)$.

\begin{tabular}{|r||rr|r|r|rrr|}
\hline & $\operatorname{id}_{M}$ & $\mu$ & $\rho$ & $\lambda$ & $\operatorname{id}_{Y^{\prime}}$ & $\delta$ & $\delta^{2}$ \\
\hline \hline $\operatorname{id}_{M}$ & $\operatorname{id}_{M}$ & $\mu$ & $\rho$ &. &. &. &. \\
$\mu$ & $\mu$ &. &. &. &. &. &. \\
\hline$\rho$ &. &. &. &. & $\rho$ &. &. \\
\hline$\lambda$ & $\lambda$ &. & $\delta^{2}$ &. &. &. &. \\
\hline $\operatorname{id}_{Y^{\prime}}$ &. &. &. & $\lambda$ & $\operatorname{id}_{Y^{\prime}}$ & $\delta$ & $\delta^{2}$ \\
$\delta$ &. &. &. &. & $\delta$ & $\delta^{2}$ &. \\
$\delta^{2}$ &. &. &. &. & $\delta^{2}$ &. &. \\
\hline
\end{tabular}

Thus we deduce that $\left.M_{\mathbb{F}_{2}}^{\prime}\right|_{\mathcal{S}_{n-2}} \cong\left[\begin{array}{c}1_{\mathbb{F}_{2}} \\ 1_{\mathbb{F}_{2}}\end{array}\right] \oplus D^{[n-3,1]}$, implying that $\left.M_{\mathbb{F}_{2}}\right|_{\mathcal{S}_{n-2}} \not$ $\left.M_{\mathbb{F}_{2}}^{\prime}\right|_{\mathcal{S}_{n-2}}$, the final contradiction to the assumption $(*)$.

Hence there is a $1_{\mathbb{F}_{2}}$-local submodule $L_{0}<Y_{\mathbb{F}_{2}}$ such that $L_{0}^{\prime}<L_{0}$, thus $L_{0}^{\prime}$ and $L_{0}$ are the only $1_{\mathbb{F}_{2}}$-local submodules, and we conclude that $\operatorname{rad}\left(L_{0}\right)=$ $\operatorname{soc}\left(Y_{\mathbb{F}_{2}}\right)=L_{0}^{\prime} \oplus L_{2}^{\prime}$, finally completing the case $n \equiv 3(\bmod 4)$.

\section{The natural module $D^{[n-1,1]}$}

We finally prove (1.2) and (1.4) revealing the structure of the tensor square $D^{[n-1,1]} \otimes D^{[n-1,1]}$, for the cases $p=2$ and $p$ odd, respectively.

(5.1) The case $p$ odd. For $n \equiv 0(\bmod p)$ the assertion follows directly from $(2.3),(2.2)$ and $(2.6)$. For $n \not \equiv 0(\bmod p)$ we by $(2.6)$ have $D^{[n-1,1]} \cong S_{\mathbb{F}_{2}}^{[n-1,1]} \cong$ $Y_{\mathbb{F}_{2}}^{[n-1,1]}$, hence $D^{[n-1,1]} \otimes D^{[n-1,1]}$ is a direct sum of Young modules, and thus the assertion again follows from (2.2).

(5.2) The case $p=2$ and $n$ odd. Since $D^{[n-1,1]} \cong S_{\mathbb{F}_{2}}^{[n-1,1]} \cong Y_{\mathbb{F}_{2}}^{[n-1,1]}$ is a Young module, $D^{[n-1,1]} \otimes D^{[n-1,1]}$ is a direct sum of Young modules as well, and hence $D^{[n-1,1]} \otimes D^{[n-1,1]} \cong Y_{\mathbb{F}_{2}}^{[n-1,1]} \oplus Y_{\mathbb{F}_{2}}^{\left[n-2,1^{2}\right]} \cong D^{[n-1,1]} \oplus Y_{\mathbb{F}_{2}}^{\left[n-2,1^{2}\right]}$.

(5.3) The case $p=2$ and $n$ even. We first consider the case $n=4$. Since $D^{[3,1]}$ has the Klein 4-group $V_{4} \triangleleft \mathcal{S}_{4}$ in its kernel, $D^{[3,1]}$ is a module for $\mathcal{S}_{4} / V_{4} \cong$ $\mathcal{S}_{3}$, and as such isomorphic to $D^{[2,1]}$. The module $Y_{\mathbb{F}_{2}}^{\left[1^{3}\right]} \cong\left[\begin{array}{c}1_{\mathbb{F}_{2}} \\ 1_{\mathbb{F}_{2}}\end{array}\right]$ is a module for $\mathcal{S}_{3} / \mathcal{A}_{3} \cong C_{2} \cong \mathcal{S}_{4} / \mathcal{A}_{4}$. Hence by $(5.2)$ we get $D^{[3,1]} \otimes D^{[3,1]} \cong D^{[3,1]} \oplus\left[\begin{array}{l}1_{\mathbb{F}_{2}} \\ 1_{\mathbb{F}_{2}}\end{array}\right] \cong$ $\operatorname{rad}\left(Y_{\mathbb{F}_{2}}^{\left[2,1^{2}\right]}\right) / \operatorname{soc}\left(Y_{\mathbb{F}_{2}}^{\left[2,1^{2}\right]}\right)$, which in particular is decomposable. 
We now let $n \geq 6$. We have $V:=Y_{\mathbb{F}_{2}}^{[n-1,1]} \otimes Y_{\mathbb{F}_{2}}^{[n-1,1]} \cong 2 \cdot Y_{\mathbb{F}_{2}}^{[n-1,1]} \oplus Y_{\mathbb{F}_{2}}^{\left[n-2,1^{2}\right]}$. Fixing a direct sum decomposition of $V$ as above, we let $\pi: V \rightarrow \operatorname{im}(\pi) \cong$ $Y_{\mathbb{F}_{2}}^{\left[n-2,1^{2}\right]}$ be the associated projection with $\operatorname{ker}(\pi) \cong Y_{\mathbb{F}_{2}}^{[n-1,1]} \oplus Y_{\mathbb{F}_{2}}^{[n-1,1]}$. From the unique composition series of $Y_{\mathbb{F}_{2}}^{[n-1,1]}$ we get a filtration

$$
V \cong\left[\frac{1_{\mathbb{F}_{2}}}{\frac{D^{[n-1,1]} \oplus D^{[n-1,1]}}{\frac{1_{\mathbb{F}_{2}} \oplus\left(D^{[n-1,1]} \otimes D^{[n-1,1]}\right) \oplus 1_{\mathbb{F}_{2}}}{D^{[n-1,1]} \oplus D^{[n-1,1]}}}}\right],
$$

hence there is $U<V$ having a filtration $\{0\}<U_{1}<U_{2}<U$ with layers

$$
U \cong\left[\frac{D^{[n-1,1]} \otimes D^{[n-1,1]}}{D^{[n-1,1]} \oplus D^{[n-1,1]}}\right] .
$$

Now $V$ contains the constituent $D^{[n-2,2]}$ with multiplicity 2 , and from the submodule structure of $\operatorname{im}(\pi) \cong Y_{\mathbb{F}_{2}}^{\left[n-2,1^{2}\right]}$ given in Table 2 we deduce that there are precisely two $D^{[n-2,2]}$-local submodules $L_{2}^{\prime}<L_{2}<\operatorname{rad}(\operatorname{im}(\pi))<V$. Since $D^{[n-1,1]} \otimes D^{[n-1,1]}$ also contains the constituent $D^{[n-2,2]}$ with multiplicity 2 we have $L_{2} \leq U$ as well. From $\operatorname{soc}(\operatorname{im}(\pi)) \cong \operatorname{soc}\left(Y_{\mathbb{F}_{2}}^{\left[n-2,1^{2}\right]}\right) \cong D^{[n-1,1]}$ we get $1_{\mathbb{F}_{2}} \cong U_{1} \leq \operatorname{ker}(\pi)$. Moreover there is a submodule $U_{1}<L \leq U_{2} \cap \operatorname{ker}(\pi)$ such that $L \sim 1_{\mathbb{F}_{2}} \oplus D^{[n-1,1]}$. Hence in conclusion we have $L \oplus L_{2} \leq U$.

We now for the moment distinguish two cases. For $n \equiv 2(\bmod 4)$ we have $D^{[n-1,1]} \otimes D^{[n-1,1]} \sim 4 \cdot 1_{\mathbb{F}_{2}} \oplus D^{[n-1,1]} \oplus 2 \cdot D^{[n-2,2]}$, hence $U \sim 5 \cdot 1_{\mathbb{F}_{2}} \oplus 3 \cdot D^{[n-1,1]} \oplus$ $2 \cdot D^{[n-2,2]}$, and by Table 2 we obtain $L_{2} \sim 3 \cdot 1_{\mathbb{F}_{2}} \oplus 2 \cdot D^{[n-1,1]} \oplus 2 \cdot D^{[n-2,2]}$. For $n \equiv 0(\bmod 4)$ we similarly get $D^{[n-1,1]} \otimes D^{[n-1,1]} \sim 2 \cdot 1_{\mathbb{F}_{2}} \oplus D^{[n-1,1]} \oplus 2 \cdot D^{[n-2,2]}$, hence $U \sim 3 \cdot 1_{\mathbb{F}_{2}} \oplus 3 \cdot D^{[n-1,1]} \oplus 2 \cdot D^{[n-2,2]}$ and $L_{2} \sim 1_{\mathbb{F}_{2}} \oplus 2 \cdot D^{[n-1,1]} \oplus 2 \cdot D^{[n-2,2]}$.

Thus in both cases we may argue as follows. We have $\operatorname{soc}\left(L_{2}\right) \cong D^{[n-1,1]}$. Assume that $\operatorname{soc}\left(L_{2}\right) \not \leq U_{2}$, then we conclude $L_{2} \cong\left(L_{2}+U_{2}\right) / U_{2} \leq U / U_{2} \cong$ $D^{[n-1,1]} \otimes D^{[n-1,1]}$, contradicting the above multiplicities. Thus $\operatorname{soc}\left(L_{2}\right)<U_{2}$, and hence from $U_{2} \sim 1_{\mathbb{F}_{2}} \oplus 2 \cdot D^{[n-1,1]}$ we deduce $U_{2}=L \oplus \operatorname{soc}\left(L_{2}\right)$, where $L=U_{2} \cap \operatorname{ker}(\pi)$. Moreover, we have $U /\left(L \oplus L_{2}\right) \cong 1_{\mathbb{F}_{2}}$. Hence $U / U_{2}$ has shape $\left[\frac{1_{\mathbb{F}_{2}}}{\bar{L}_{2}}\right]$, where $\bar{L}_{2}:=L_{2} / \operatorname{soc}\left(L_{2}\right)=L_{2} / \operatorname{soc}(\operatorname{im}(\pi))$.

Assume that $\widehat{L}:=U \cap \operatorname{ker}(\pi)>U_{2} \cap \operatorname{ker}(\pi)=L$, then since $L_{2}<\operatorname{im}(\pi)$ we have $\widehat{L} / L \cong 1_{\mathbb{F}_{2}}$ and $\widehat{L} \oplus L_{2}=U$, implying $U / U_{2} \cong\left(\widehat{L} \oplus L_{2}\right) /\left(L \oplus \operatorname{soc}\left(L_{2}\right)\right) \cong 1_{\mathbb{F}_{2}} \oplus \bar{L}_{2}$. Hence $U / U_{2} \cong D^{[n-1,1]} \otimes D^{[n-1,1]}$ has a subquotient isomorphic to $1_{\mathbb{F}_{2}} \oplus 1_{\mathbb{F}_{2}}$. We deduce that $\left.\left(U / U_{2}\right)\right|_{\mathcal{S}_{n-1}} \cong D^{[n-2,1]} \otimes D^{[n-2,1]}$ also has a subquotient isomorphic to $1_{\mathbb{F}_{2}} \oplus 1_{\mathbb{F}_{2}}$, by $(5.2)$ contradicting Table 2 . Hence we have $\widehat{L}=L$, and thus 
$U / L \cong U^{\pi}=\operatorname{rad}(\operatorname{im}(\pi)) \cong \operatorname{rad}\left(Y_{\mathbb{F}_{2}}^{\left[n-2,1^{2}\right]}\right)$. Since $U / U_{2} \cong(U / L) /\left(U_{2} / L\right) \cong$ $U^{\pi} / \operatorname{soc}\left(L_{2}\right)=\operatorname{rad}(\operatorname{im}(\pi)) / \operatorname{soc}(\operatorname{im}(\pi))$ this proves the asserted isomorphism.

For $n \equiv 2(\bmod 4)$ the module $\operatorname{rad}(\operatorname{im}(\pi)) / \operatorname{soc}(\operatorname{im}(\pi))$ is uniserial and hence indecomposable. For $n \equiv 0(\bmod 4)$ and $n \geq 6$ let $\operatorname{soc}(\operatorname{im}(\pi))<L_{1}^{\prime}<L_{1}<$ $\operatorname{rad}(\operatorname{im}(\pi))$ be the $1_{\mathbb{F}_{2}}$-local submodules of $\operatorname{im}(\pi)$, and let $\bar{L}_{1}:=L_{1} / \operatorname{soc}(\operatorname{im}(\pi))$. Fixing a direct sum decomposition of $\operatorname{rad}(\operatorname{im}(\pi)) / \operatorname{soc}(\operatorname{im}(\pi))$, there is a unique summand containing $\bar{L}_{1}$, and a unique summand containing $\bar{L}_{2}$ as a submodule. Since $\bar{L}_{1} \cap \bar{L}_{2} \neq\{0\}$ and $\bar{L}_{1}+\bar{L}_{2}=\operatorname{rad}(\operatorname{im}(\pi)) / \operatorname{soc}(\operatorname{im}(\pi))$ we conclude that $\operatorname{rad}(\operatorname{im}(\pi)) / \operatorname{soc}(\operatorname{im}(\pi))$ is indecomposable, and we are done.

\section{References}

[1] D. Benson: Representations and cohomology I, Cambridge studies in advanced mathematics 30, Cambridge Univ. Press, 1991.

[2] D. Benson: Some remarks on the decomposition numbers for the symmetric groups, in: The Arcata Conference on Representations of Finite Groups, 1986, Proc. Sympos. Pure Math. 47, part 1, 381-394, Amer. Math. Soc., 1987.

[3] D. Benson, J. Conway: Diagrams for modular lattices, J. Pure Appl. Algebra 37, 1985, 111-116.

[4] C. Bessenrodt, A. Kleshchev: On tensor products of modular representations of symmetric groups, Bull. London Math. Soc. 32, 2000, 292-296.

[5] G. Birkhoff: Lattice theory, 3rd edition, American Mathematical Society Colloquium Publications 25, Amer. Math. Soc., 1984.

[6] J. Brundan, A. KleshChev: Representations of the symmetric group which are irreducible over subgroups, J. Reine Angew. Math. 530, 2001, $145-190$.

[7] C. Curtis, I. Reiner: Methods of representation theory, vol. I, with applications to finite groups and orders, reprint of the 1981 original, Wiley Classics Library, Wiley, 1990.

[8] K. ERdmann: Young modules for symmetric groups, Special issue on group theory, J. Aust. Math. Soc. 71, 2001, 201-210.

[9] W. FeIT: The representation theory of finite groups, North-Holland, 1982.

[10] The GAP Group: GAP-4.4 - Groups, Algorithms, and Programming, 2006, http://www.gap-system.org.

[11] R. Gow, A. Kleshchev: Connections between the representations of the symmetric group and the symplectic group in characteristic 2, J. Algebra 221, 1999, 60-89.

[12] J. Graham, G. James: On a conjecture of Gow and Kleshchev concerning tensor products, J. Algebra 227, 2000, 767-782.

[13] J. Grabmeier: Unzerlegbare Moduln mit trivialer Young-Quelle und Darstellungstheorie der Schur-Algebra, Bayreuther Math. Schr. 20, 1985, 9-152. 
[14] A. Henke: On $p$-Kostka numbers and Young modules, Eur. J. Combin. 26, 2005, 923-942.

[15] A. Henke: Schur subalgebras and an application to the symmetric group, J. Algebra 233, 2000, 342-362.

[16] G. James: Trivial source modules for symmetric groups. Arch. Math. 41, 1983, 294-300.

[17] G. James: Representations of the symmetric groups over the field of order 2, J. Algebra 38, 1976, 280-308.

[18] G. James: The representation theory of the symmetric group, Lecture Notes in Mathematics 682, Springer, 1978.

[19] G. James, A. Kerber: The representation theory of the symmetric group, Encyclopedia of Mathematics 16, Adddison-Wesley, 1981.

[20] A. KleshChev: Linear and projective representations of symmetric groups, Cambridge Tracts in Mathematics 163, Cambridge Univ. Press, 2005.

[21] K. Lux, J. Müller, M. Ringe: Peakword condensation and submodule lattices: an application of the MeatAxe, J. Symb. Comput. 17, 1994, 529544.

[22] K. Lux, M. SzőKE: Computing homomorphism spaces between modules over finite dimensional algebras, Experiment. Math. 12, 2003, 91-98.

[23] S. Martin: Schur algebras and representation theory, Cambridge Tracts in Mathematics 112, Cambridge Univ. Press, 1993.

[24] A. Mathas: Iwahori-Hecke algebras and Schur algebras of the symmetric group, University Lecture Series 15, Amer. Math. Soc., 1999.

[25] J. MüLleR: On a theorem by Benson and Conway, J. Pure Appl. Algebra 208, 2007, 89-100.

[26] J. MÜller, R. Zimmermann: Green vertices and sources of simple modules of the symmetric group labelled by hook partitions, Arch. Math. 89, 2007, 97-108.

[27] M. Ringe: The C-MeatAxe 2.4, RWTH Aachen, 2003.

[28] J. ORLoB: Zerlegung von Tensorprodukten einfacher Moduln der symmetrischen Gruppe, Diplomarbeit, RWTH Aachen, 2006.

[29] M. PEEL: Hook representations of the symmetric groups, Glasgow Math. J. 12, 1971, 136-149.

[30] J. ShEтH: Branching rules for two row partitions and applications to the inductive systems for symmetric groups, Comm. Algebra, 27, 1999, 33033316.

[31] M. SzŐKE: Examining Green correspondents of simple modules, Dissertation, RWTH Aachen, 1998.

Lehrstuhl D Für Mathematik, RWTH Aachen

Templergraben 64, D-52062 Aachen, Germany

Juergen.Mueller@math.rwth-aachen.de

Johannes.Orlob@math.rwth-aachen.de 